\title{
The Planetary Nebula Luminosity Function of M87 and the Intracluster Stars of Virgo
}

\author{
Robin Ciardullo ${ }^{1,2}$ \\ Department of Astronomy and Astrophysics, Penn State University, 525 Davey Lab, \\ University Park, PA 16802 \\ George H. Jacoby \\ Kitt Peak National Observatory, National Optical Astronomy Observatories, P.O. Box \\ 26732, Tucson, AZ 85726 \\ and \\ John J. Feldmeier and Roger E. Bartlett \\ Department of Astronomy and Astrophysics, Penn State University, 525 Davey Lab, \\ University Park, PA 16802
}

Received —

\footnotetext{
${ }^{1}$ Visiting Astronomer, Kitt Peak National Optical Astronomy Observatories, which is operated by the Association of Universities for Research in Astronomy, Inc., under cooperative agreement with the National Science Foundation.

${ }^{2} \mathrm{NSF}$ Young Investigator
} 


\begin{abstract}
We present the results of a wide-field [O III] $\lambda 5007$ survey for planetary nebulae $(\mathrm{PN})$ in M87 and its surrounding halo. In all, we identify $338 \mathrm{PN}$ candidates in a $16^{\prime} \times 16^{\prime}$ field around the galaxy; 187 of these objects are in a statistical sample which extends to $m_{5007}=27.15$. We show that the planetary nebula luminosity function (PNLF) of M87's halo is unlike any PNLF observed to date, with a shape that differs from that of the empirical law at the $99.9 \%$ confidence level. In addition, we find that the PNLF of M87's outer halo differs from that of the galaxy's inner regions at a high degree of certainty $(\sim 92 \%)$. We show that both these effects are most likely due to the existence of intracluster PN, many of which are foreground to M87. These intracluster objects explain the "overluminous" [O III] $\lambda 5007$ sources previously identified by Jacoby, Ciardullo, \& Ford (1990), and present us with a new tool with which to probe the morphological and dynamical properties of the cluster.

By modifying the maximum likelihood procedures of Ciardullo et al. (1989a) to take into account the presence of "field objects," and using an assumed M31 distance of 770 Kpc (Freedman \& Madore 1990) with a Burstein \& Heiles (1984) reddening law, we derive a distance modulus to M87 of $30.79 \pm 0.16$ $(14.4 \pm 1.1 \mathrm{Mpc})$. This value is in excellent agreement with the previous survey of Jacoby, Ciardullo, \& Ford (1990) and contradicts the assertion of Bottinelli et al. (1991) and Tammann (1992) that the PNLF distance to Virgo has been underestimated due to inadequate survey depth.
\end{abstract}

Subject headings: galaxies: distances - galaxies: individual (M87) - galaxies: — nebulae: planetary — galaxies: clusters (Virgo) — intergalactic medium 


\section{Introduction}

The planetary nebula luminosity function (PNLF) technique is one of the simplest methods for determining extragalactic distances out to $\sim 20 \mathrm{Mpc}$. One takes a deep exposure of a galaxy through a filter which passes light at [O III] $\lambda 5007$, and compares the image to a slightly deeper exposure off the emission line. Those stellar objects that appear

on the [O III] $\lambda 5007$ image but not on the off-band frame are either planetary nebulae (PN) or compact H II regions. If the target object is a normal elliptical or S0 galaxy with no star formation, then the presence of H II regions can generally be discounted, and one is left with a list of $\mathrm{PN}$, from which one can form a luminosity function.

The power of the PNLF technique comes from the shape of the luminosity function. At faint magnitudes, the PNLF has the power law form predicted from models of uniformly expanding shells surrounding slowly evolving central stars (Henize \& Westerlund 1963; Jacoby 1980). However, observations have demonstrated that the bright end of the PN luminosity function dramatically breaks from this relation, and falls to zero very quickly, within $\sim 0.7 \mathrm{mag}$ (cf. Jacoby et al. 1992). It is the constancy of this cutoff magnitude, $M^{*}$, and its high monochromatic luminosity, that makes the PNLF such a useful standard candle.

The shape and absolute magnitude of the PNLF cutoff has been successfully reproduced theoretically by a number of authors, including Jacoby (1989), Dopita, Jacoby, \& Vassiliadis (1992), Méndez et al. (1993), Han, Podsiadlowski, \& Eggleton (1994), and Richer, McCall, \& Arimoto (1997). Nevertheless, Bottinelli et al. (1991) and Tammann (1992) have argued that the bright-end of the PNLF is actually a power law, and thus observations which do not reach the break in the luminosity function are not useful for distance measurements. In support of this model, Bottinelli et al. and Tammann point to the "overluminous" [O III] $\lambda 5007$ sources found by Jacoby, Ciardullo, \& Ford (1990) in Virgo elliptical galaxies, which 
can plausibly be argued to be part of a high-luminosity tail to the PNLF. By adopting the power-law model, and ignoring evidence for curvature in the observed PNLF of Virgo, Bottinelli et al. and Tammann have argued that the PNLF distance to this cluster is biased towards too low a value. Although Kolmogorov-Smirnov and $\chi^{2}$ tests show this interpretation is highly unlikely, the most unambiguous way to test the hypothesis is to perform a deep, wide-field [O III] $\lambda 5007$ imaging survey of the Virgo ellipticals and better define the shape of the faint-end of the PNLF.

In this paper, we report the results of a $16^{\prime} \times 16^{\prime}[\mathrm{O}$ III $] \lambda 5007$ survey centered on the central elliptical of Virgo, M87. In $\S 2$, we give the details of the survey, present the positions and magnitudes of 338 planetary nebulae found in the galaxy's envelope and outer halo, and estimate the photometric accuracy of our measurements by comparing our derived PN magnitudes with those given in Jacoby, Ciardullo, \& Ford (1990). In §3, we select two statistically complete subsets of these planetaries, and demonstrate that our planetary nebula luminosity function extends well onto the Henize \& Westerlund (1963) tail, making a distance determination to the galaxy possible. In $\S 4$, we discuss the surprising result that the PNLF of M87's outer halo has a cutoff that is $\sim 0.2 \mathrm{mag}$ brighter than that for the inner part of the galaxy. We then show that, in retrospect, this behavior could have been predicted, since the intracluster stars of Virgo should produce a considerable number of planetary nebulae, and some of these objects will be foreground to M87. In $\S 7$, we include this "field" contribution in our maximum likelihood analysis, and derive a distance to M87 of $14.4 \pm 1.1 \mathrm{Mpc}$, in good agreement with the previous PNLF distance determination to the galaxy. This result vitiates the hypothesis of Bottinelli et al. (1991) and Tammann (1992) that the bright-end of the PNLF is an unbounded power-law. We conclude by discussing the implications intracluster PN have for morphological and dynamical studies of nearby clusters. 


\section{Observations and Reductions}

On 6 and 7 April 1995 we surveyed a 16' $\times 16^{\prime}$ region of sky around M87 with the T2KB CCD on the Kitt Peak 4-m telescope, which afforded a plate scale of 0 ". 47 per pixel. Our on-band data consisted of seven exposures totaling 6.8 hours through a $\sim 30 \AA$ full-width-half-maximum (FWHM) interference filter centered at $5030 \AA$ in the converging $\mathrm{f} / 2.7$ beam of the telescope. (The transmission curve of this filter at the ambient temperature of $11^{\circ} \mathrm{C}$ is displayed in Figure 1.) Our off-band data was composed of five 540 sec exposures through a $267 \AA$ wide filter centered at $5312 \AA$. In addition, an H $\alpha$ image, consisting of nine 900 sec exposures through a $75 \AA$ FWHM interference filter centered at $6606 \AA$, was obtained on 8 April 1995. These latter data were used to discriminate PN from compact H II regions, supernova remnants, and emission associated with M87's cooling flow. The seeing for our $\lambda 5007$ on-band survey was 1.'2; our $\mathrm{H} \alpha$ data was taken in better than 1."0 seeing.

Planetary nebula candidates were identified and measured in a manner similar to that described in detail by Jacoby et al. (1989), Ciardullo, Jacoby, \& Ford (1989b), and Jacoby, Ciardullo, \& Ford (1990). After spatially registering all the individual frames, we combined the on-band, off-band, and $\mathrm{H} \alpha$ frames of each field, using the imcombine task in IRAF to reject radiation events. We then "blinked" the on-band [O III] $\lambda 5007$ sum against the off-band $\lambda 5312$ and $\mathrm{H} \alpha$ sum. Objects clearly visible on the on-band image, but absent on the off-band and $\mathrm{H} \alpha$ frame were noted as possible planetaries. We confirmed these identifications by examining the candidates on each individual on-band frame, and then looking closely at the appearance of each object on our [O III] $\lambda 5007$ "difference" picture.

Equatorial coordinates for the PN candidates were derived using 86 reference stars from the USNO-A.1.0 Astrometric Catalog (Monet 1996) to define the CCD's coordinate system; the internal error in these coordinates is $\sim 0$ ". 5 . [O III] $\lambda 5007$ photometry was 
accomplished relative to bright field stars with the DAOPHOT point-spread-function fitting routines (Stetson 1987) within IRAF. These measurements were placed on the standard system by comparing large aperture measurements of the field stars with similar measurements of the Stone (1977) and Oke (1974) spectrophotometric standards G191B2B, Feige 34, BD+25 3941, and BD+40 4032. The dispersion in the photometric zero point computed from these stars was 0.03 mag. Finally, we computed the standard $\lambda 5007$ magnitudes for the PN by modeling the filter transmission curve (Jacoby et al. 1989) and using the photometric procedures for emission-line objects described by Jacoby, Quigley, \& Africano (1987). For this computation, the systemic velocity of M87 was taken from the Third Reference Catalog of Bright Galaxies (de Vaucouleurs et al. 1991), and the galaxy's envelope velocity dispersion was estimated from Sargent et al. (1978). Note that since the systemic velocity of M87 is near the peak of the filter transmission curve, a $\sim 100 \mathrm{~km} \mathrm{~s}^{-1}$ error in the latter quantity translates into a flux error of only $\sim 0.03$ mag.

Table 1 lists the PN candidates identified in the field of M87, and follows on from the numbering scheme of Jacoby, Ciardullo, \& Ford (1990). Columns 2, 3, and 4 of the table list the objects' epoch 2000 coordinates and $\lambda 5007$ magnitudes as defined by Ciardullo et al. (1989a),

$$
m_{5007}=-2.5 \log F_{5007}-13.74
$$

Column 5 gives the semi-major axis of the isophote upon which each PN is superposed. For $r_{\text {iso }}<5$ ! 8 , these values were determined using the surface photometry of Cohen (1986); at larger distances, the isophotal radii were computed from an assumed axis ratio $b / a=0.77$ and a galactic position angle of p.a $=158^{\circ}$. Table 2 lists an additional 9 PN that are projected very near other galaxies in the field and are presumably bound to them. These objects are included only for completeness and are not used in any of our analyses.

Table 3 gives the mean errors in our photometric measurements as reported by the 
PSF-fitting algorithms of DAOPHOT. However, because portions of M87 have been previously surveyed by Jacoby, Ciardullo, \& Ford (1990), it is possible to independently assess our errors by comparing the two data sets. Of the $55 \mathrm{PN}$ identified by Jacoby et al., 45 were recovered in this survey. A comparison of the magnitudes of the four PN with the highest signal-to-noise ratio shows that there is no statistical difference between the magnitude system of the two surveys: the zero point of the new observations is $0.03 \pm 0.06$ mag brighter than that from 1990. However, as Figure 2 demonstrates, a comparison of the entire dataset indicates that there is an additional source of scatter $\sim 0.1 \mathrm{mag}$ above that expected from the combined errors of the two measurements. Part of the scatter is probably due to differences in filter transmission curves, as M87's internal velocity dispersion will shift the emission lines of some objects on the filters' wings. (We correct for this effect in the mean using the techniques outlined in Jacoby et al. (1989) and Ciardullo, Jacoby, \& Ford (1989b), but corrections for individual objects cannot be made without velocity information. Most of the additional error probably comes from the 1990 data, which was compromised by variable seeing and a high readout noise RCA CCD. Nevertheless, for the analysis below, we have added an additional 0.07 mag uncertainty in quadrature to the errors listed in Table 3. In practice, the amplitude of the error term makes very little difference to our final results.

Nine PN candidates from Jacoby, Ciardullo, \& Ford (1990) were not detected in this survey: PN \# 30, 35, 45, 49, 50, 51, 52, 53, and 54. In addition, PN candidate \# 55 also was not recovered, but it fell at the position of a CCD defect, and thus could not be checked. Eight of these objects were at the limit of the previous survey and below the stated completeness limit; the other two were near the limit of completeness. All of the brighter PN from the previous survey were easily recovered in this new, wide-field survey. 


\section{The PNLF of M87's Halo}

Figure 3 displays the raw planetary nebula luminosity function for M87. As is illustrated, our data extend well past the PNLF cutoff onto the expected power-law tail. Although these data are not statistically complete, and cannot be used for a precise distance estimate, it is clear that the position of the PNLF cutoff is much brighter than the $m_{5007} \sim 27.0$ value needed if the Virgo core is at the distance suggested by Sandage \& Tammann $(1995,1996)$.

To form a statistical sample of PN, we began by considering the detectability of planetary nebulae in our field. For most of our $16^{\prime} \times 16^{\prime}$ survey region, the background sky (which is the dominant source of photometric noise) is constant, hence the limiting magnitude for PN detections is constant. However, near the center of M87, the galaxy background dominates, and the detectability of faint PN decreases. To address this problem, we used the results of Ciardullo et al. (1987) and Hui et al. (1993), who showed that PN detections are essentially 100\% complete when the DAOPHOT signal-to-noise ratio (SNR) is greater than $\sim 9$, but no PN are detected when the SNR is less than $\sim 4$. Thus, for our statistical sample, we included only those PN with a DAOPHOT measurement error of $\sigma_{\text {err }}<1.086 / \mathrm{SNR}=0.12 \mathrm{mag}$. At isophotal galactic radii greater than $2^{\prime}$, this completeness criteria included essentially all PN brighter than $m_{5007}=27.2$. At galactic radii smaller than this, however, the limiting magnitude decreased quickly, so that by $r_{\text {iso }}<0$.' 5 , no PN were detectable. We therefore defined our "complete sample" to be those PN with $m_{5007}<27.15$ and $r_{\text {iso }}>2^{\prime}$. 


\section{The Planetary Nebula Luminosity Function of M87}

Figure 4 plots the planetary nebula luminosity function for the statistical sample of PN. From these data, the PNLF distance to the galaxy can normally be derived by convolving the empirical model for the PNLF given by Ciardullo et al. (1989a)

$$
N(M) \propto e^{0.307 M}\left[1-e^{3\left(M^{*}-M\right)}\right]
$$

with the photometric error function (Table 3) and fitting the data to the resultant curve via the method of maximum likelihood. However, in the case of M87, the most likely empirical curve is a poor fit to the luminosity function (cf. Figure 4). In fact, Kolmogorov-Smirnov and $\chi^{2}$ tests both exclude the Ciardullo et al. law at the $99.9 \%$ confidence interval. This is a remarkable result: none of the PNLFs from any of the $\sim 30$ previously studied galaxies differs significantly from the empirical law. Moreover, the large number of PN detected in this survey cannot be invoked to explain the discrepancy. The luminosity functions of M31 (Ciardullo et al. 1989a), M81 (Jacoby et al. 1989), NGC 5128 (Hui et al. 1993), and NGC 4494 (Jacoby, Ciardullo, \& Harris 1996) all contain similar numbers of objects. The planetaries surrounding M87 are therefore unique in some way.

An even more surprising result comes if we divide our PN sample in two, and compare the PNLFs of M87's inner and outer halo. For the inner sample (sample "A"), we take all the PN in our statistical sample with isophotal radii between $2^{\prime}$ and $4^{\prime}$; for the outer sample, (sample "B") we take those PN with $r_{\text {iso }}>4^{\prime}$. Both samples are plotted in Figure 5. As is illustrated, sample "A" contains PN \#1, the extremely overluminous object first identified by Jacoby, Ciardullo, \& Ford (1990). However, of the 20 brightest PN in the samples, 18 belong to sample "B". More importantly, the shapes of the two PNLFs appear different: a Kolmogorov-Smirnov test reveals that the two samples are different at the $92 \%$

confidence level. Again, this result is unique. Explicit tests for changes in the PNLF cutoff with galactocentric radius have been performed with the large samples of PN available in 
NGC 5128 (224 objects; Hui et al. 1993) and NGC 4494 (101 objects; Jacoby, Ciardullo, \& Harris 1996). In neither case was a gradient observed.

\section{Explaining the Luminosity Function}

Internal and external tests on the $\sim 30$ early and late-type galaxies surveyed to date have shown that the PNLF cutoff is remarkably insensitive to changes in stellar population (cf. Jacoby 1996). However, a number of mechanisms do exist which can, at least in theory, cause the PNLF technique to fail and produce a change in the observed value of $m^{*}$. The first, and simplest, is to hypothesize that some instrumental effect exists, such as a radial gradient in the flatfield or the transmission curve of the filter. We have examined the former possibility by comparing the flatfields taken through our [O III] $\lambda 5007$ Virgo filter with similar flats taken through a different (lower redshift) [O III] $\lambda 5007$ filter that same night (cf. Feldmeier, Ciardullo, \& Jacoby 1997). No large-scale difference is apparent. Similarly, we tested for problems with the filter bandpass by tracing the transmission curve of our filter at four different positions on the glass: to within $\sim 3 \AA$, the central wavelength of our filter is the same at all points. Since at the velocity of M87, a $\sim 3 \AA$ shift in wavelength corresponds to, at most, a $\sim 3 \%$ change in the filter transmission (cf. Figure 1), the effect is much too small to explain our result.

A second method for explaining the variation in $m^{*}$ is to invoke non-uniform extinction in the galaxy. Dust has been detected in the central regions of many elliptical galaxies (e.g., Sadler \& Gerhard 1985; Ebneter, Djorgovski, \& Davis 1988; Goudfrooij et al. 1994), and several authors have suggested that extinction is responsible for the presence of radial color gradients in these systems (Witt, Thronson, \& Capuano 1992; Goudfrooij \& De Jong 1995; Wise \& Silva 1996). However, these studies have concentrated on the central regions of elliptical galaxies, while our survey deals exclusively with PN that are more than 1.5 
effective radii $\left(r_{e}\right)$ from the galactic nucleus. In fact, there is little reason to believe that the extinction in M87 changes by $E(B-V) \sim 0.05$ between 2 and $4 r_{e}$. Moreover, even if there is a strong gradient in the dust distribution, this still may not translate into an observed gradient in $m^{*}$. As Feldmeier, Ciardullo, \& Jacoby (1997) have shown, the location of a galaxy's PNLF cutoff is relatively insensitive to the presence of dust, as long as the scale length for the obscuration is smaller than that of the stars. (This is because the bright end of the PNLF (i.e., the PNLF cutoff) is always dominated by the bright, unextincted PN. Unless the dust extends far enough to cover virtually all the PN, the bright-end cutoff will always consist of unreddened objects.) This makes it very unlikely that dust is responsible for the change in $m^{*}$.

Although the absolute magnitude of the PNLF cutoff is extremely insensitive to the details of the underlying stellar population, a dramatic change in the metallicity or age of M87's halo stars could, in principle, produce a change in $m^{*}$ similar to that observed. Both observations (Ciardullo \& Jacoby 1992; Richer 1994) and theory (Dopita, Jacoby \& Vassiliadis 1992) suggest that galaxies with $[\mathrm{O} / \mathrm{H}] \lesssim-0.5$ can have a PNLF cutoff that is different from that of metal-rich populations by $\sim 0.1$ mag. Unfortunately, this effect acts in the wrong direction: it is the metal-poor systems that have fainter values of $M^{*}$. In order to explain the observed gradient, the center of M87 would have to be metal-poor, and the halo would need to be metal-rich. Observations show that this is extremely unlikely (Kormendy \& Djorgovski 1989).

Similarly, it is difficult to use population age to explain PNLF variations. According to the models of Dopita, Jacoby, \& Vassiliadis (1992) and Méndez et al. (1993), the location of the PNLF cutoff is nearly independent of age for populations between 3 and 12 Gyr. If these models are correct, then in order to enhance the luminosity of the PNLF cutoff in M87's outer halo, one must hypothesize an unrealistically young age for the stars, $\sim 0.5$ Gyr. 
Moreover, even this may not be sufficient, as there is excellent agreement between the PNLF and Cepheid distances to the Large Magellanic Cloud and M101 (Jacoby, Walker, \& Ciardullo 1990; Feldmeier, Ciardullo, \& Jacoby 1997). These observations strongly suggest that the location of the PNLF cutoff does not change by much, even in systems with active star formation. It is therefore difficult to attribute changes in the PNLF cutoff to variations in stellar population.

\section{Planetary Nebulae and the Intracluster Stars of Virgo}

The best hope for explaining the observed changes in M87's halo PNLF lies in the Virgo Cluster itself. All of the above explanations implicitly assume that the PN projected onto M87's outer halo are at the same distance as those PN which are members of the inner sample. However, if the Virgo Cluster has a substantial population of intracluster stars, this

will not be the case, as some objects will be superposed in the foreground, and others will be in the background. For example, if the Virgo Cluster is at a distance of $\sim 15 \mathrm{Mpc}$, then the central $6^{\circ}$ core of the cluster (de Vaucouleurs 1961), has a linear extent of $\sim 1.5 \mathrm{Mpc}$. If the core is spherically symmetric and filled with stars, then we might expect some intracluster objects to be up to $\sim 0.25$ mag brighter than the value of $m^{*}$ derived from galaxies at the center of the cluster. This is roughly what is observed in Figure 5.

Further evidence that the anomalous PNLF of M87 is due to foreground contamination comes from the fact that it is the outer sample of objects that has most of the bright PN. The number of foreground PN detected in any region of our CCD field should be roughly proportional to the area of the field; since the outer region samples $\sim 8$ times more area than the inner field, those data should contain $\sim 8$ times more intracluster objects. In addition, M87's sharply peaked surface brightness profile guarantees that the ratio of galaxy light to intracluster light in the inner field will be much larger than that in the outer field. 
Consequently, the contribution of intracluster objects to sample "A" will be small, while that for sample "B" will be relatively large. Again, this is roughly what is displayed in Figure 5 .

The intracluster PN hypothesis also explains the existence of "overluminous" planetary nebulae. In their survey of Virgo, Jacoby, Ciardullo, \& Ford (1990) identified a small number of [O III] $\lambda 5007$ sources that were significantly brighter than predicted by the empirical planetary nebula luminosity function. As discussed by Jacoby, Ciardullo, \& Harris (1996), there are a number of possible origins for these objects, including compact H II regions, supernova remnants, Wolf-Rayet nebulae, supersoft x-ray nebulae, coalesced binary-star nebulae, chance superpositions of multiple objects, and even background quasars at $z=3.1$. However, most of these explanations are not satisfactory from a stellar population standpoint, and the remaining ideas have little observational support. In fact, to investigate this question, we imaged the overluminous object NGC 4406 PN \#1 (Jacoby, Ciardullo, \& Ford 1990) with the Planetary Camera of the Hubble Space Telescope. Even at WFPC II resolution, the object is unresolved. Since $\sim 0$ "'05 at Virgo corresponds to a linear size of $\sim 4 \mathrm{pc}$, this observation effectively excludes H II regions, supernova remnants, and PN superpositions from the list of possibilities.

The existence of intracluster PN provides a natural explanation for the overluminous planetaries. It also explains why similarly bright [O III] $\lambda 5007$ sources have not been detected in the Leo I, Triangulum, Coma I, or Fornax Clusters, nor in any field galaxy. If the overluminous PN are indeed intracluster in origin, then isolated field galaxies will be clean systems without foreground contaminants. Furthermore, of the clusters observed to date, only Fornax is rich enough to have a substantial intracluster environment, but it has very little front-to-back depth (Tonry 1991; McMillan, Ciardullo, \& Jacoby 1993). Virgo is the only cluster surveyed for PN where depth of field is important, and it is the only cluster 
in which overluminous planetaries are found.

Although the idea of intracluster planetary nebulae seems speculative, there is, in fact, conclusive evidence that such objects do exist. In their radial velocity survey of $19 \mathrm{PN}$ in the halo of the Virgo Cluster elliptical M86 $\left(v=-220 \mathrm{~km} \mathrm{~s}^{-1}\right)$, Arnaboldi et al. (1996) found three objects with $v>1300 \mathrm{~km} \mathrm{~s}^{-1}$. These planetaries are undoubtably intracluster in origin. Significantly, the PN observed by Arnaboldi et al. were originally identified with a $30 \AA$ filter centered at $5007 \AA$ (Jacoby, Ciardullo, \& Ford 1990); intracluster objects with $v>1000 \mathrm{~km} \mathrm{~s}^{-1}$ should have been strongly excluded. The only reason three were detected was that at $v \sim 1500 \mathrm{~km} \mathrm{~s}^{-1}$, [O III] $\lambda 4959$ is shifted into the bandpass of the [O III] $\lambda 5007$ filter! Since $I(\lambda 5007) / I(\lambda 4959)=3$, only the very brightest PN could have been detected in this way. The existence of three intracluster objects in the Arnaboldi et al. sample therefore implies the presence of many more.

Similar evidence for a population of intragalactic stars in Virgo comes from SN 1980I. This event occurred in intergalactic space, midway between the Virgo core ellipticals NGC 4374 and NGC 4406. Considering that only $~ 12$ probable SN Ia supernovae have occurred in the cluster core this century (Barbon, Cappallaro, \& Turatto 1989), the existence of one intergalactic object suggests that intracluster stars must be fairly common.

Intracluster PN have also been proven to exist in the Fornax Cluster. An imaging survey of three intergalactic fields by Theuns \& Warren (1997) turned up ten planetary nebula candidates in $104 \mathrm{sq}$. arcmin of sky. When this detection rate is extrapolated to the entire cluster, the result is that a substantial fraction of the Fornax Cluster's stars, perhaps as much as $\sim 40 \%$, must be in intergalactic space. If the same is true for Virgo, then the effect of intracluster objects on the galactic PNLFs cannot be ignored.

In fact, a quantitative estimate for the stellar mass of Virgo's intracluster environment comes from observations of faint stars by the Hubble Space Telescope. By comparing the 
number counts of objects in a Virgo field with that in the Hubble Deep Field, Ferguson, Tanvir, \& Von Hippel (1997) found an excess of $\sim 600$ stars in a 4.6 square arcmin region. If these are red giant and asymptotic giant branch stars, and if their mean lifetime at this luminosity is $\sim 10^{6}$ years, then a simple scaling of areas and lifetimes implies the existence of $\sim 700 \mathrm{PN}$ in our survey field. If, as implied by equation $(2), \sim 2 \%$ of these PN are bright enough to be observable, then our PNLF of M87 should be contaminated by $\sim 14$ intracluster objects. Given the uncertainties involved, this is in agreement with our data.

\section{The Distance to M87}

The presence of intragalactic planetary nebulae in the Virgo Cluster impedes our ability to measure M87's distance via the planetary nebula luminosity function technique. Of the two samples of PN plotted in Figure 5, clearly sample "A" is the better one to use for this purpose, but even it has some contamination from intracluster objects. This "field" contribution must be removed before any distance measurement to the galaxy can be attempted.

To do this, we first made the assumption that all PN with isophotal radii $r>4^{\prime} 8$ $\left(r>3 r_{e}\right)$ are intracluster in origin. (This is probably not strictly true; bound PN have been found $\sim 3.7 r_{e}$ from the center of NGC 3379 (Ciardullo, Jacoby, \& Dejonghe 1993) and more than $\sim 4 r_{e}$ away from the nucleus in NGC 5128 (Hui et al. 1995). However, this radius represents the best compromise between the need to exclude galactic objects and the desire for good counting statistics.) We then binned our sample of intracluster PN (sample "C") into 0.2 mag intervals, and fit a smooth curve through the points. This curve, displayed in Figure 6, represents our estimate of the intracluster PN luminosity function.

With the field luminosity function in hand, we proceeded to derive an expression for 
the most likely distance of M87. If we treat both the empirical PNLF of equation (2) and the intracluster PNLF as probability distributions, then in any magnitude range, $d m$, the expected number of observable $\mathrm{PN}$ is

$$
\lambda(m) d m=[T \epsilon \circ \phi(m-\mu)+\xi \beta(m)] d m
$$

where $\epsilon \circ \phi(M)$ is the convolution of the photometric error function (Table 3) with the (normalized) empirical luminosity function, $\beta(m)$ is the (normalized) intracluster luminosity function, $T$ is the total population of M87 planetaries in our field, and $\xi$ is the total number of intracluster PN in our field. Following Hanes \& Whittaker (1987) and Ciardullo et al. (1989a), the relative probability of observing a given set of $N$ planetaries down to limiting magnitude $m_{l}$, as a function of distance modulus $\mu, T$, and $\xi$, is

$$
\ln P(T, \mu, \xi)=-\int_{-\infty}^{m_{l}} T \phi(m-\mu) d m-\int_{-\infty}^{m_{l}} \xi \beta(m) d m+\sum_{i}^{N} \ln \{T \phi(m-\mu)+\xi \beta(m)\}
$$

Now note that, although $\xi$ is an independent variable, it is constrained by the observations. If $N_{f}$ is the number of intracluster PN in sample "C", and $A$ is the ratio of the area of sample "A" to the area of sample "C", then $\xi$ should be distributed according to the Poisson distribution function, $P_{P}$, with a mean of $N_{f} A=11.1$ and a standard deviation of $\sqrt{N_{f}} A=1.25$. Thus, when integrated over all $T$ and $\mu$, equation (4) should give

$$
\iint P(T, \mu, \xi) d T d \mu=P_{P}(\xi)
$$

This renormalization of (4) enabled us to derive a distance to M87 despite the fact that the functions $\phi(M)$ and $\beta(m)$ are somewhat similar in shape. The formulation also maintains the advantage of the original PNLF maximum-likelihood technique, in that it avoids all problems associated with the binning of small amounts of data.

Figure 7 displays the likelihood of each solution as a function of M87's distance modulus. For the plot, we have assumed a foreground Galactic extinction of $E(B-V)=0.023$ 
(Burstein \& Heiles (1984), a Seaton (1979) reddening law, and a value of $M^{*}=-4.54$ based on an M31 distance of 770 kpc (Freedman \& Madore 1990) and an M31 extinction of $E(B-V)_{\mathrm{M} 31}=0.08$ (Burstein \& Heiles 1984). The most likely distance modulus is $(m-M)_{0}=30.79(14.4 \mathrm{Mpc})$; the formal uncertainty of the result is $+0.07 \mathrm{mag},-0.06 \mathrm{mag}$. This distance would decrease by $\sim 3 \%$ if the Ciardullo et al. (1989a) value of $M^{*}(-4.48)$ were used.

The present result is in excellent agreement with the previous PNLF distance determination. When scaled to the same set of assumptions regarding the PNLF zero point and Galactic extinction, the Jacoby, Ciardullo, \& Ford (1990) value for M87 is $(m-M)_{0}=30.85 \pm 0.09(14.8 \pm 0.6 \mathrm{Mpc})$. Note that the previous survey was limited by a small sample size (only 36 objects in the statistical sample) and a noisy CCD chip. It did, however, extend to smaller galactocentric radii than the present survey, and thus was not as badly contaminated by intracluster objects. Consequently, although the present survey contains many more PN and has a greater photometric accuracy, the distance derivation is not significantly better than the older work.

An interesting feature of our new PNLF distance measurement is the robustness of the result. Because the empirical PNLF of equation (2) goes to zero at $m<m^{*}$, the earlier PNLF measurements in Virgo were sensitive to the luminosity of the brightest one or two planetaries. In fact, as Jacoby, Ciardullo, \& Ford (1990) pointed out, the inclusion (or exclusion) of overluminous objects from their Virgo samples could change the derived distances to galaxies by more than $\sim 20 \%$. (Jacoby et al. handled this problem by noting that overluminous PN could be excluded based on the overall quality of the fits - solutions that included overluminous objects were significantly poorer than fits without them.) The present analysis, however, is much less sensitive to the existence of these objects. For example, if PN \#1 is arbitrarily excluded from our sample, the derived distance 
to M87 changes by less than 0.02 mag. Figure 6 plots the best fit luminosity function, $T \phi(m-\mu)+\xi \beta(m)$. As is illustrated, the fit is excellent.

The agreement between the two PNLF distance estimates strongly refutes the contention of Bottinelli et al. (1991) and Tammann (1992) that sample size affects PNLF distance measurements. The new measurement uses over a factor of two more planetaries than the older determination, yet the distance to the galaxy remains substantially the same. Moreover, the presence of intracluster stars in Virgo negates one of the core tenets of Tammann (1992), that overluminous PN are a natural extension of the empirical PNLF. This interpretation is no longer needed to explain the data.

The distance error quoted above is the formal uncertainty derived from our maximum likelihood procedure, not the total error in the computed distance. To obtain the latter quantity, the uncertainty in the solution must first be combined with usual random uncertainties associated with the photometric zero point (0.03 mag), the correction associated with going from the DAOPHOT magnitudes to the large aperture magnitudes (0.02 mag), the filter calibration (0.03 mag), and the Galactic extinction (0.05 mag). In addition, the present analysis has an additional error, that associated with the uncertain shape of the field PN luminosity function. Simulations suggest that this error is small, probably less than $\sim 0.05$ mag. Thus, the total random uncertainty in our distance determination is $\sim 0.11$ mag.

Finally, two sources of error that affect all PNLF measurements is the uncertainty in the distance to M31, which provides the zero point $(0.10 \mathrm{mag})$, and the definition of the empirical PNLF itself (0.05 mag). The addition of these two quantities in quadrature yields a total uncertainty of 0.16 mag.

Our new distance modulus to M87, $(m-M)_{0}=30.79 \pm 0.16(14.4 \pm 1.1 \mathrm{Mpc})$ is consistent with distances to the galaxy derived from the surface brightness fluctuation 
method (30.92 \pm 0.12; Ciardullo, Jacoby, \& Tonry 1993, Tonry 1997), and the globular cluster luminosity function (31.12 \pm 0.26 ; Whitmore et al. 1995). It is, however, marginally smaller than the median distance of $16.1 \pm 1.0 \mathrm{Mpc}$ obtained from Cepheid measurements in the Virgo Cluster (Pierce et al. 1994; Ferrarese et al. 1996; Sandage et al. 1996; Saha et al. 1996a,b). This difference may not be significant; our results point out the problem with using Cepheids to estimate the distance to the Virgo Cluster core. The brightest PN in sample "C" is $\sim 0.35$ mag brighter than the value of $m^{*}$ in M87. This implies that the Virgo Cluster core extends at least 2.1 Mpc in depth. Furthermore, of the five spirals with Cepheid measurements, only three are actually projected within the $6^{\circ}$ core, and only NGC 4571 (which has a ground-based Cepheid distance of $14.9 \pm 1.2 \mathrm{Mpc}$ ) shows any evidence of being physically in the core (cf. van der Hulst et al. 1987). Thus, the issue of cluster depth cannot be neglected.

Nevertheless, our PNLF measurements should give a reliable estimate of the distance to the Virgo core. Although M87 is offset by about $1^{\circ}$ from the center of the cluster's isopleths, and has a radial velocity that is $\sim 200 \mathrm{~km} \mathrm{~s}^{-1}$ larger than the cluster mean (Binggeli, Tammann, \& Sandage 1987), the galaxy is almost certainly at the center of the cluster's potential. Velocity and positional offsets, such as those observed for M87, are common in cD galaxies within dynamically young clusters (Bird 1994), and there is good evidence to suggest that Virgo is such a cluster. Based on the radial velocities of dwarf galaxies inside the Virgo Cluster core, Binggeli, Popescu, \& Tammann (1993) have concluded that there is a separate group of galaxies associated with M86 (NGC 4406) that is falling into Virgo from the far side of the cluster. Independent confirmation of this hypothesis comes from Jacoby, Ciardullo, \& Ford (1990), whose PNLF distances place M86 (radial velocity $-220 \mathrm{~km} \mathrm{~s}^{-1}$ ) over $1 \mathrm{Mpc}$ behind M87. If this interpretation is correct, then the masses of M86 and M87, as derived from their x-ray halos, imply that the peculiar position and velocity of M87 is a direct consequence of the disturbance caused by the infall of M86 and its group (Böhringer 
et al. 1994).

In fact, the best evidence of M87's position within the Virgo Cluster comes from the x-ray data obtained by the ROSAT satellite. The distribution of Virgo's intracluster x-ray gas demonstrates quite conclusively that a large portion of the cluster's mass is centered at the position M87 (Böhringer et al. 1994). This fact, along with the presence of a diffuse cD halo around the galaxy (cf. Weil, Bland-Hawthorn, \& Malin 1997), strongly indicates that M87 is, indeed, at the center of the cluster. Our distance measurement to M87 should therefore be representative of that of the group.

\section{Planetary Nebulae as Probes of the Virgo Cluster}

The existence of intracluster planetary nebulae provides us with a new and unique tool for probing the structure of Virgo. For example, Huchra $(1985,1988)$ has pointed out that, from isopleths and velocity measurements alone, it is difficult to tell whether or not the central $6^{\circ}$ core of Virgo is virialized. Evidence for virialization includes the nearly Gaussian distribution of velocities for the early-type galaxies, and the excellent agreement between the apparent morphology of the core and distribution of galaxies expected from an isothermal King (1962) model; evidence against virialization comes from the positional and velocity offset between the x-ray halo of M87 and the cluster center. Thus, while Huchra (1988) states that the Virgo ellipticals form an apparently virialized core, Binggeli, Tammann, \& Sandage (1987), and Binggeli, Popescu, \& Tammann (1993) argue strongly that the cluster contains significant substructure, even near its center.

The observed planetary nebula luminosity function can help answer this question. Old stellar populations produce planetary nebulae in proportion to the population's total

luminosity (Renzini \& Buzzoni 1986; Ciardullo 1995). Hence the number of intergalactic 
PN detected in our survey should reflect the total amount of intracluster luminosity present in our field. More importantly, since the observed PNLF is formed from a superposition of PNLFs at different distances along the line-of-sight, the precise shape of the observed luminosity function contains information on the cluster's front-to-back morphology. It is therefore possible to test models of the Virgo Cluster by comparing the field PNLF of Figure 6 with the luminosity distributions expected from different cluster profiles.

Figure 8 demonstrates this possibility. In the figure, we compare the field PNLF of M87 (sample "C") with the PNLF expected from an isothermal cluster with a core radius of $r_{c}=0.5 \mathrm{Mpc}$ and a center $52^{\prime}$ from the position of our survey field (Binggeli, Tammann, \& Sandage 1987). It is obvious that the model fails to fit the bright end of the PNLF by many orders of magnitude. In fact, this result is a general property of all dynamically relaxed cluster profiles: once virialization occurs, the cluster becomes much too condensed to explain the large number of (presumably) foreground PN present in the observed PNLF. To come close to producing the requisite number of bright objects, the radial profile of the cluster must be shallower. However, as Figure 8 shows, even a uniform density law fails to fit the overall shape of observed PNLF. Consequently, it is likely that the distribution of intergalactic stars in Virgo is clumpy, and that the cluster is not virialized.

Since planetary nebulae sample the light, it is possible, at least in theory, to use our observations to place a constraint on the fraction of the Virgo Cluster's dark matter that is in intergalactic stars. In practice, however, three difficulties prevent us from using our data in this manner. First is the limitation imposed by our narrow-band filter. The interference filter used for this project was designed to detect the PN associated with M87, and its $30 \AA$ FWHM bandpass is ideal for this project (cf. Figure 1). However, the Virgo Cluster core has an observed velocity dispersion of $\sigma_{v} \approx 800 \mathrm{~km} \mathrm{~s}^{-1}$ (Binggeli, Tammann, \& Sandage 1987), or $\sim 26 \AA$ at the wavelength of the [O III] $\lambda 5007$ emission line. As a result, our 
survey is probably missing a significant number of intracluster planetaries, perhaps as many as $30 \%$.

A second difficulty with the interpretation of our data is that our CCD field is not at a "typical" location in the Virgo Cluster, but is instead centered on M87. Consequently, the density of intracluster objects in our field is likely to be higher than average. Moreover, we cannot exclude the possibility that some of the PN at large galactocentric radii are actually bound to the M87. While it may be possible to remove this contribution by extrapolating the luminosity profile of M87 out to large radii, this profile is not very well known (cf. Weil, Bland-Hawthorn, \& Malin 1997; Graham et al. 1996). Furthermore, any extrapolation would require the extra assumption that M87's luminosity-specific PN number density does not change with radius. Unfortunately, this quantity has been observed to increase with galactocentric radius in NGC 5128 (Hui et al. 1993) and in a composite galaxy formed from eight elliptical galaxies (Ciardullo, Jacoby, \& Feldmeier 1995). Thus, the validity of this assumption is unknown.

By far the most serious obstacle encountered when trying to use our PN detections to estimate the density of Virgo's intracluster stars comes from the finite depth of the cluster and the steeply rising bright-end of the PNLF. Our [O III] $\lambda 5007$ survey reached a limiting magnitude of $m_{5007}=27.15$; thus, if the distance to the Virgo Cluster core is $\sim 15 \mathrm{Mpc}$ and its size is $\sim 1.5 \mathrm{Mpc}$, then our measurements sample the top $\sim 1$ mag of the PNLF on the front side of the cluster. On the back-side of Virgo, however, our [O III] $\lambda 5007$ measurements only go $\sim 0.5$ mag down the luminosity function. In terms of equation (2), this means that the intracluster luminosity in the foreground of Virgo receives $\sim 2.6$ times more weight than luminosity on the backside. If the radial profile of the Virgo Cluster were smooth, this effect, as well as the others presented above, could be modeled. However, since it is likely that sub-structure is present in the core of Virgo, any extrapolation of the results 
from our one CCD field to the entire cluster is premature.

\section{Conclusion}

We have detected 338 planetary nebulae in our new, wide-field survey of M87 and its surrounding halo. The analysis of the luminosity function of these PN demonstrates that M87 is at a distance of $14.4 \pm 1.1 \mathrm{Mpc}$; this number is in excellent agreement with the earlier PNLF measurement, as well as recent distance determinations from the surface brightness fluctuation method and the globular cluster luminosity function. The result is, however, in sharp disagreement with the hypothesis of Bottinelli et al. (1991) and Tammann (1992) that PNLF measurements in Virgo are biased due to a limited sample size.

Our observations of M87 also present strong evidence for the presence of a substantial population of intergalactic stars, which extends over $\sim 2 \mathrm{Mpc}$ in front of M87. The intergalactic stars are, in all likelihood, the explanation for the "overluminous" planetaries encountered by Jacoby, Ciardullo, \& Ford (1990) in Virgo, but not seen any where else (Jacoby, Ciardullo \& Harris 1996). The analysis of the intracluster PN luminosity function suggests that the Virgo Cluster core is not virialized, but is instead dynamically young. However, the mismatch between the width of our [O III] $\lambda 5007$ filter and the Virgo Cluster velocity dispersion precludes a definitive statement or a more detailed analysis at the present time.

Planetary nebula observations offer a new opportunity for morphological and dynamical studies of nearby clusters. To date, the only way to study intergalactic light in clusters has been through deep surface photometry, and as a result, only a few, very rich, Abell Clusters have been measured (cf. Uson, Boughn, \& Kuhn 1991; Vílchez-Gómez, Pelló, \& Sanahuja 1994). Planetary nebula observations offer an alternative to these studies, 
and provide information on both the two and three dimensional structure of the cluster. Moreover, a PN's [O III] $\lambda 5007$ emission line is an excellent target for a radial velocity measurement. Since there are never enough sufficiently bright galaxies in a cluster to fully define the cluster's velocity field, planetary nebulae can provide invaluable data for cluster dynamics. Future [O III] $\lambda 5007$ surveys may therefore allow us to probe the effects of galactic mergers, cluster accretion, and tidal-stripping within several nearby clusters, and enable new investigations into the distribution of dark matter in clusters and of the initial conditions of cluster formation.

We would like to thank Mike Pierce for first suggesting the possible intracluster origin of some of M87's halo PN. This work was supported in part by NASA grant NAGW-3159 and NSF grant AST-9529270. Support for this work was also partially provided by NASA through grant number GO-0612.01-94A from the Space Telescope Science Institute, which is operated by the Association of Universities for Research in Astronomy, Inc., under NASA contract NAS5-26555. 


\section{REFERENCES}

Arnaboldi, M., Freeman, K.C., Mendez, R.H., Capaccioli, M., Ciardullo, R., Ford, H., Gerhard, O., Hui, X., Jacoby, G.H., Kudritzki, R.P., \& Quinn, P.J. 1996, ApJ, 472, 145

Barbon, R., Cappallaro, E., \& Turatto, M. 1989, A\&AS, 81, 421

Binggeli, B., Popescu, C.C., \& Tammann, G.A. 1993, A\&AS, 98, 275

Binggeli, B., Tammann, G.A., \& Sandage, A. 1987, AJ, 94, 251

Bird, C.M. 1994, AJ, 107, 1637

Böhringer, H., Briel, U.G., Schwarz, R.A., Voges, W., Hartner, G., \& Trümper, J. 1994, Nature, 368, 828

Bottinelli, L., Gouguenheim, L., Paturel, C., \& Teerikorpi, P. 1991, A\&A, 252, 550

Burstein, D., \& Heiles, C. 1984, ApJS, 54, 33

Ciardullo, R. 1995, IAU Highlights of Astronomy, 10, ed. I. Appenzeller (Dordrecht: Kluwer), p. 507

Ciardullo, R., Ford, H.C., Neill, J.D., Jacoby, G.H., \& Shafter, A.W. 1987, ApJ, 318, 520

Ciardullo, R., \& Jacoby, G.H. 1992, ApJ, 388, 268

Ciardullo, R., Jacoby, G.H., \& Dejonghe, H.B. 1993, ApJ, 414, 454

Ciardullo, R., Jacoby, G.H., \& Tonry, J.L. 1993, ApJ, 419, 479

Ciardullo, R., Jacoby, G., \& Feldmeier, J. 1995, BAAS, 26, 1400

Ciardullo, R., Jacoby, G.H., \& Ford, H.C. 1989b, ApJ, 344, 715 
Ciardullo, R., Jacoby, G.H., Ford, H.C., \& Neill, J.D. 1989a, ApJ, 339, 53

Cohen, J.G. 1986, AJ, 92, 1039

de Vaucouleurs, G. 1961, ApJS, 6, 213

de Vaucouleurs, G., de Vaucouleurs, A., Corwin, H.G. Jr., Buta, R.J., Paturel, G., \& Fouqué, P. 1991, Third Reference Catalog of Bright Galaxies (Springer-Verlag, New York)

Dopita, M.A., Jacoby, G.H., \& Vassiliadis, E. 1992, ApJ, 389, 27

Ebneter, K., Djorgovski, S., \& Davis, M. 1988, AJ, 95, 422

Feldmeier, J.J., Ciardullo, R., \& Jacoby, G.H. 1997, ApJ, 479, 231

Ferguson, H., Tanvir, N., \& von Hippel, T. 1997, Nature, in press

Ferrarese, L., Freedman, W.L., Hill, R.J., Saha, A., Madore, B.F., Kennicutt, R.C., Jr., Stetson, P.B., Ford, H.C., Graham, J.A., Hoessel, J.G., Han, M., Huchra, J., Hughes, S.M., Illingworth, G.D., Kelson, D., Mould, J.R., Phelps, R., Silbermann, N.A., Sakai, S., Turner, A., Harding, P., \& Bresolin, F. 1996, ApJ, 464, 568

Freedman, W.L., \& Madore, B.F. 1990, ApJ, 365, 186

Goudfrooij, P., \& De Jong, T. 1995, A\&A, 298, 784

Graham, A., Lauer, T.R., Colless, M., \& Postman, M. 1996, ApJ, 465, 534

Goudfrooij, P., Hansen, L., Jörgensen, H.E., \& Norgaard-Nielsen, H.U., 1994, A\&AS, 105, 341

Han, Z., Podsiadlowski, P., \& Eggleton, P.P. 1994, MNRAS, 270, 121

Hanes, D.A., \& Whittaker, D.G. 1987, AJ, 94, 906 
Henize, K.G., \& Westerlund, B.E. 1963, ApJ, 137, 747

Huchra, J.P. 1988, in ASP Conf. Ser. \#4, The Extragalactic Distance Scale, ed. by S. van den Bergh \& C.J. Pritchet (Astronomical Society of the Pacific, Provo), p. 257

Huchra, J.P. 1985, in ESO Workshop on the Virgo Cluster of Galaxies, ed. by O. Richter \& B. Binggeli (ESO, Garching), p. 181

Hui, X., Ford, H., Ciardullo, R., \& Jacoby, G. 1993, ApJ, 414, 463

Hui, X., Ford, H., Freeman, K.C., \& Dopita, M.A. 1995, ApJ, 449, 592

Jacoby, G.H. 1980, ApJS, 42, 1

Jacoby, G.H. 1989, ApJ, 339, 39

Jacoby, G.H. 1996, IAU Symposium 180, Planetary Nebulae, ed. by H. Habing \& H. Lamers (Kluwer, Dordrecht), in press

Jacoby, G.H., Branch, D., Ciardullo, R., Davies, R.L, Harris, W.E., Pierce, M.J., Pritchet, C.J., Tonry, J.L., \& Welch, D.L. 1992, PASP, 104, 599

Jacoby, G.H., Ciardullo, R., \& Ford, H.C. 1990, ApJ, 356, 332

Jacoby, G.H., Ciardullo, R., Ford, H.C., \& Booth, J. 1989, ApJ, 344, 704

Jacoby, G.H., Ciardullo, R., \& Harris, W.E. 1996, ApJ, 462, 1

Jacoby, G.H., Quigley, R.J., \& Africano, J.L. 1987, PASP, 99, 672

Jacoby, G.H., Walker, A.R., \& Ciardullo, R. 1990, ApJ, 365, 471

King, I.R. 1962, AJ, 67, 471

Kormendy, J., \& Djorgovski, S. 1989, ARA\&A, 27, 235 
McMillan, R., Ciardullo, R., \& Jacoby, G.H. 1993, ApJ, 416, 62

Méndez, R.H., Kudritzki, R.P., Ciardullo, R., \& Jacoby, G.H. 1993, A\&A, 275, 534

Monet, D. 1996, BAAS, 28, 905

Oke, J.B. 1974, ApJS, 27, 21

Pierce, M.J., Welch, D.L., McClure, R.D., van den Bergh, S., Racine, R., \& Stetson, P.B. 1994, Nature, 371, 385

Renzini, A., \& Buzzoni, A. 1986, in Spectral Evolution of Galaxies, ed. C. Chiosi, \& A. Renzini (Dordrecht: Reidel), p. 195

Richer, M.G. 1994, Ph.D. Thesis, York University

Richer, M.G., McCall, M.L., \& Arimoto, N. 1997, A\&A, in press

Sadler, E.M., \& Gerhard, O.E. 1985, MNRAS, 214, 177

Saha, A., Sandage, A., Labhardt, L., Tammann, G.A., Macchetto, F.D., \& Panagia, N. 1996a, ApJ, 466, 55

Saha, A., Sandage, A., Labhardt, L., Tammann, G.A., Macchetto, F.D., \& Panagia, N. 1996b, ApJS, 107, 693

Sandage, A., \& Tammann, G.A. 1995, ApJ, 446, 1

Sandage, A., \& Tammann, G.A. 1996, ApJ, 464, 51

Sandage, A., Saha, A., Tammann, G.A., Labhardt, L., Panagia, N., \& Macchetto, F.D. 1996, ApJ, 460, L15

Sargent, W.L.W., Young, P.J., Boksenberg, A., Shortridge, K., Lynds, C.R., \& Hartwick, F.D.A. 1978, ApJ, 221, 731 
Seaton, M.J. 1979, MNRAS, 187, 73p

Stetson, P.B. 1987, PASP, 99, 191

Stone, R.P.S. 1977, ApJ, 218, 767

Tammann, G.A. 1992, in IAU Symposium 155, Planetary Nebulae, ed. by R. Weinberger \& A. Acker (Kluwer, Dordrecht), p. 515

Theuns, T., \& Warren, S.J. 1997, MNRAS, 284, L11

Tonry, J.L. 1991, ApJ, 373, L1

Tonry, J.L. 1997, private communication

Uson, J.M., Boughn, S.P., \& Kuhn, J.R. 1991, ApJ, 369, 46

van der Hulst, J.M., Skillman, E.D., Kennicutt, R.C., \& Bothun, G.D. 1987, A\&A, 177, 63

Vílchez-Gómez, R., Pelló, R., \& Sanahuja, B. 1994, A\&A, 283, 37

Weil, M.L., Bland-Hawthorn, J., \& Malin, D.F. 1997, MNRAS, in press

Whitmore, B.C., Sparks, W.B., Lucas, R.A., Macchetto, F.D., \& Biretta, J.A. 1995, ApJ, 454, L73

Wise, M.W., \& Silva, D.R. 1996, ApJ, 461, 155

Witt, A.N., Thronson, H.A., Jr., \& Capuano, J.M., Jr. 1992, ApJ, 393, 611 
Fig. 1. - The transmission curve of our [O III $] \lambda 5007$ filter when placed in the $\mathrm{f} / 2.7$ beam of the Kitt Peak 4-m telescope at the outside temperature of $11^{\circ} \mathrm{C}$. Also shown is the $\pm 2 \sigma$ velocity dispersion of M87's envelope (from Sargent et al. 1978) and the $\pm 2 \sigma$ velocity dispersion of the Virgo Cluster (from Binggeli, Tammann, \& Sandage 1987).

Fig. 2.- A comparison of the PN [O III $] \lambda 5007$ magnitudes of this survey with those obtain by Jacoby, Ciardullo, \& Ford (1990). The dotted lines define the internal $1 \sigma$ photometric error derived in DAOPHOT. Although the data suggest that DAOPHOT underestimates the true photometric error by as much as $\sim 0.1$, some of the additional scatter can be attributed to differences in the transmission curves. Most of the outliers lie on areas are projected on areas of high galaxy background, where the uncertainty in determining the sky level dominates.

Fig. 3.- The luminosity function for our entire set of planetary nebulae around M87 binned into 0.1 mag intervals. Although the data do not represent a statistical sample, it is obvious that the data reach past the cutoff to the power-law tail of the faint-end of the luminosity function. It is also clear that for $M^{*}=-4.5$, the distance modulus of $(m-M) \sim 31.5$ advocated by Sandage \& Tammann $(1995 ; 1996)$ is incompatible with the data.

Fig. 4.- The planetary nebula luminosity function for a sample of planetaries with isophotal radii greater than $2^{\prime}$ binned into 0.2 mag intervals. The error bars show the $1 \sigma$ uncertainty of counting statistics, and the open circle represents the place where incompleteness is becoming important. The curve shows the empirical function shifted to the most likely distance modulus. Although this curve is the "best fit" to the data, it is still excluded at the $99.9 \%$ confidence level.

Fig. 5.- The open squares show the planetary nebula luminosity function for PN with isophotal radii between $2^{\prime}$ and $4^{\prime}$. The solid circles show a similar dataset, but for objects 
with $r_{\text {iso }}>4^{\prime}$. Both sets of data have been binned into 0.2 mag intervals and have the error bars that represent the $1 \sigma$ uncertainties of counting statistics. Note that although the brightest $\mathrm{PN}$ is part of the inner sample, the vast majority of bright objects are found at large galactocentric radii. In fact, the hypothesis that both sets of data are drawn from the same underlying distribution is excluded at the $92 \%$ confidence level.

Fig. 6. - The circles show the planetary nebula luminosity function for a complete set of M87 planetaries with $m_{5007}<27.15$ and isophotal radii between $2^{\prime}$ and $4^{\prime}$; the squares show the PNLF for intracluster objects scaled to the same area of the galactic survey. The $1 \sigma$ uncertainties due to Poisson statistics are shown by error bars, and the open square and circle denote data past the limit of completeness. The dotted curve represents a smooth fit to the intracluster PNLF, while the solid curve shows the most likely galactic plus intergalactic luminosity function. Note that this combined luminosity function fits the observed PNLF very well.

Fig. 7.- The results of the maximum likelihood analysis for M87. The abscissa is the true distance modulus; the ordinate is the relative probability that the observed PNLF is drawn from a combination of the intracluster "field" PNLF and the empirical model (Ciardullo et al. 1989a) at the given distance. In the plot, we have assumed a differential extinction $E(B-V)=0.023$ and a Seaton (1979) reddening law.

Fig. 8. - The luminosity function of intracluster planetary nebulae compared to two cluster models. The solid curve gives the PNLF expected from an isothermal cluster with core radius 1.7 and center at $\alpha(1950)=12^{\mathrm{h}} 15^{\mathrm{m}}, \delta(1950)=+13^{\circ}$ (Binggeli, Tammann, \& Sandage 1987). Note that this model is much too condensed to fit the data. A better model, displayed by the dotted line, is one in which the intracluster PN are assumed to have a uniform density and extend $\sim 4 \mathrm{Mpc}$ from M87, but even this law is not an adequate representation of the data. 
Table 1. Planetary Nebulae Sample

\begin{tabular}{ccccc}
\hline \hline & & & & \\
ID & $\alpha(2000)$ & $\delta(2000)$ & $m_{5007}$ & $R_{\text {iso }}$ \\
\hline & & & & \\
1 & 123049.38 & 122058.5 & 25.63 & 2.58 \\
2 & 123052.45 & 122113.5 & 26.11 & 2.37 \\
3 & 123045.49 & 122201.5 & 26.16 & 1.92 \\
4 & 123046.08 & 121937.9 & 26.05 & 4.28 \\
5 & 123043.87 & 122039.2 & 26.58 & 3.58 \\
6 & 123049.69 & 122204.3 & 26.62 & 1.42 \\
7 & 123051.55 & 121940.7 & 26.35 & 3.89 \\
8 & 123047.58 & 122125.7 & 26.48 & 2.22 \\
9 & 123052.85 & 122510.0 & 26.45 & 2.17 \\
10 & 123047.98 & 122112.1 & 27.03 & 2.43 \\
11 & 123050.47 & 122006.3 & 26.60 & 3.45 \\
12 & 123046.96 & 122518.9 & 26.64 & 1.95 \\
13 & 123050.20 & 122509.0 & 26.41 & 1.81 \\
14 & 123047.03 & 122505.2 & 27.12 & 1.73 \\
15 & 123056.52 & 122415.0 & 26.62 & 2.35 \\
16 & 123054.75 & 122052.0 & 26.98 & 2.92 \\
17 & 123046.94 & 122435.9 & 26.92 & 1.28 \\
18 & 123053.18 & 122354.0 & 26.72 & 1.19 \\
19 & 123054.28 & 122002.1 & 27.12 & 3.63 \\
20 & 123044.42 & 122347.3 & 26.92 & 1.32 \\
21 & 123051.48 & 121953.8 & 26.58 & 3.66 \\
22 & 123056.40 & 122125.8 & 26.94 & 2.73 \\
23 & 123054.51 & 122214.6 & 26.97 & 1.80
\end{tabular}


Table 1-Continued

\begin{tabular}{cccccc}
\hline \hline & & & & & \\
ID & $\alpha(2000)$ & $\delta(2000)$ & $m_{5007}$ & $R_{\text {iso }}$ \\
\hline & & & & \\
24 & 123054.51 & 122214.6 & 26.97 & 1.80 \\
25 & 123043.85 & 122123.8 & 27.10 & 2.86 \\
26 & 123053.27 & 122119.2 & 26.94 & 2.35 \\
27 & 123054.69 & 122406.3 & 26.43 & 1.72 \\
28 & 123056.37 & 121905.9 & 26.95 & 4.69 \\
29 & 123042.99 & 122429.5 & 26.72 & 1.93 \\
31 & 123047.74 & 122443.1 & 26.53 & 1.32 \\
32 & 123050.79 & 122116.6 & 27.09 & 2.25 \\
33 & 123048.22 & 122144.5 & 27.24 & 1.83 \\
46 & 123046.67 & 122318.5 & 25.92 & 0.69
\end{tabular}


Table 1-Continued

\begin{tabular}{|c|c|c|c|c|}
\hline ID & $\alpha(2000)$ & $\delta(2000)$ & $m_{5007}$ & $R_{\text {iso }}$ \\
\hline 57 & 123051.41 & 122259.0 & 25.94 & 0.72 \\
\hline 58 & 123107.02 & 122002.9 & 25.97 & 5.97 \\
\hline 59 & 123030.88 & 122152.1 & 26.00 & 6.18 \\
\hline 60 & 123038.96 & 122813.8 & 26.01 & 5.42 \\
\hline 61 & 123035.04 & 122414.1 & 26.10 & 4.31 \\
\hline 62 & 123047.36 & 121840.5 & 26.12 & 5.13 \\
\hline 63 & 123044.84 & 122329.7 & 26.13 & 1.18 \\
\hline 64 & 123042.14 & 122913.9 & 26.21 & 6.05 \\
\hline 65 & 123043.21 & 122415.1 & 26.21 & 1.77 \\
\hline 66 & 123036.87 & 122617.6 & 26.22 & 4.39 \\
\hline 67 & 123043.48 & 122331.8 & 26.22 & 1.58 \\
\hline 68 & 123034.76 & 122021.2 & 26.23 & 5.97 \\
\hline 69 & 123057.55 & 121923.2 & 26.25 & 4.55 \\
\hline 70 & 123057.91 & 122142.0 & 26.25 & 2.89 \\
\hline 71 & 123018.61 & 122203.2 & 26.26 & 9.81 \\
\hline 72 & 123036.12 & 122726.6 & 26.27 & 5.27 \\
\hline 73 & 123036.24 & 122657.5 & 26.29 & 4.92 \\
\hline 74 & 123043.03 & 122245.7 & 26.29 & 1.97 \\
\hline 75 & 123052.01 & 122330.8 & 26.29 & 0.72 \\
\hline 76 & 123108.64 & 122122.5 & 26.30 & 5.98 \\
\hline 77 & 123102.92 & 123002.9 & 26.30 & 8.71 \\
\hline 78 & 123046.10 & 122752.7 & 26.31 & 4.56 \\
\hline 79 & 123027.42 & 122216.7 & 26.31 & 7.05 \\
\hline
\end{tabular}


Table 1-Continued

\begin{tabular}{|c|c|c|c|c|}
\hline ID & $\alpha(2000)$ & $\delta(2000)$ & $m_{5007}$ & $R_{\text {iso }}$ \\
\hline 80 & 123055.50 & 121544.4 & 26.31 & 7.96 \\
\hline 81 & 123053.52 & 122206.8 & 26.32 & 1.71 \\
\hline 82 & 123101.77 & 122108.1 & 26.32 & 4.17 \\
\hline 83 & 123111.24 & 122117.5 & 26.32 & 6.74 \\
\hline 84 & 123048.24 & 121833.8 & 26.39 & 5.18 \\
\hline 85 & 123051.24 & 122753.5 & 26.39 & 4.79 \\
\hline 86 & 123058.09 & 122332.6 & 26.40 & 2.57 \\
\hline 87 & 123037.15 & 121908.1 & 26.41 & 6.36 \\
\hline 88 & 123042.44 & 122300.8 & 26.42 & 2.02 \\
\hline 89 & 123039.67 & 122425.9 & 26.44 & 2.83 \\
\hline 90 & 123102.62 & 122556.1 & 26.46 & 5.25 \\
\hline 91 & 123031.32 & 123004.7 & 26.46 & 8.05 \\
\hline 92 & 123106.64 & 122441.6 & 26.47 & 5.73 \\
\hline 93 & 123052.29 & 122337.5 & 26.49 & 0.83 \\
\hline 94 & 123100.83 & 122701.1 & 26.50 & 5.61 \\
\hline 95 & 123046.76 & 122040.3 & 26.50 & 3.11 \\
\hline 96 & 123045.61 & 122335.9 & 26.51 & 0.96 \\
\hline 97 & 123038.43 & 122303.1 & 26.51 & 3.34 \\
\hline 98 & 123035.41 & 122445.5 & 26.52 & 4.24 \\
\hline 99 & 123035.21 & 122616.6 & 26.52 & 4.78 \\
\hline 100 & 123107.63 & 121731.9 & 26.52 & 7.60 \\
\hline 101 & 123103.73 & 121950.2 & 26.52 & 5.31 \\
\hline 102 & 123020.30 & 122611.8 & 26.53 & 8.86 \\
\hline
\end{tabular}


Table 1-Continued

\begin{tabular}{cccccc}
\hline \hline & & & & & \\
ID & $\alpha(2000)$ & $\delta(2000)$ & $m_{5007}$ & $R_{\text {iso }}$ \\
\hline & & & & & \\
103 & 123054.65 & 122607.8 & 26.53 & 3.46 \\
104 & 123107.42 & 122430.1 & 26.53 & 5.89 \\
105 & 123054.57 & 122338.5 & 26.55 & 1.49 \\
106 & 123102.52 & 122711.3 & 26.55 & 6.14 \\
107 & 123045.43 & 122614.1 & 26.55 & 2.94 \\
108 & 123041.77 & 122338.6 & 26.56 & 2.09 \\
109 & 123040.50 & 122520.8 & 26.56 & 2.99 \\
110 & 123044.44 & 122306.4 & 26.56 & 1.39 \\
111 & 123104.31 & 122701.9 & 26.57 & 6.47 \\
112 & 123052.28 & 121745.2 & 26.59 & 5.87 \\
123 & 123052.66 & 122316.1 & 26.64 & 0.90
\end{tabular}


Table 1-Continued

\begin{tabular}{|c|c|c|c|c|}
\hline ID & $\alpha(2000)$ & $\delta(2000)$ & $m_{5007}$ & $R_{\text {iso }}$ \\
\hline 126 & 123052.47 & 122538.3 & 26.64 & 2.59 \\
\hline 127 & 123041.00 & 122133.8 & 26.64 & 3.42 \\
\hline 128 & 123059.99 & 122123.0 & 26.64 & 3.59 \\
\hline 129 & 123033.62 & 122642.3 & 26.65 & 5.37 \\
\hline 130 & 123045.34 & 122531.3 & 26.65 & 2.28 \\
\hline 131 & 123102.98 & 122148.4 & 26.66 & 4.27 \\
\hline 132 & 123051.70 & 122209.8 & 26.66 & 1.42 \\
\hline 133 & 123019.85 & 122506.5 & 26.66 & 8.90 \\
\hline 134 & 123110.32 & 122619.2 & 26.66 & 7.62 \\
\hline 135 & 123046.45 & 122249.7 & 26.68 & 1.01 \\
\hline 136 & 123050.43 & 122606.7 & 26.69 & 2.85 \\
\hline 137 & 123033.19 & 122401.4 & 26.70 & 4.88 \\
\hline 138 & 123111.63 & 121625.1 & 26.70 & 9.13 \\
\hline 139 & 123049.25 & 121846.7 & 26.70 & 4.89 \\
\hline 140 & 123044.78 & 122257.2 & 26.71 & 1.37 \\
\hline 141 & 123047.65 & 122436.3 & 26.71 & 1.22 \\
\hline 142 & 123106.39 & 122354.7 & 26.71 & 5.39 \\
\hline 143 & 123052.70 & 122411.2 & 26.73 & 1.25 \\
\hline 144 & 123053.74 & 122653.7 & 26.73 & 4.08 \\
\hline 145 & 123107.38 & 122141.0 & 26.73 & 5.56 \\
\hline 146 & 123053.87 & 122402.0 & 26.73 & 1.46 \\
\hline 147 & 123040.06 & 122525.1 & 26.74 & 3.14 \\
\hline 148 & 123044.04 & 122830.5 & 26.74 & 5.24 \\
\hline
\end{tabular}


Table 1-Continued

\begin{tabular}{ccccc}
\hline \hline & & & & \\
ID & $\alpha(2000)$ & $\delta(2000)$ & $m_{5007}$ & $R_{\text {iso }}$ \\
\hline & & & & \\
149 & 123048.66 & 122234.6 & 26.74 & 0.91 \\
150 & 123037.55 & 122954.5 & 26.74 & 7.06 \\
151 & 123052.92 & 122350.2 & 26.74 & 1.08 \\
152 & 123021.78 & 121639.3 & 26.75 & 2.00 \\
153 & 123059.73 & 121916.4 & 26.75 & 4.95 \\
154 & 123026.18 & 122359.8 & 26.75 & 7.02 \\
155 & 123046.85 & 122043.2 & 26.75 & 3.05 \\
156 & 123041.93 & 122648.6 & 26.76 & 3.82 \\
157 & 123051.15 & 122226.7 & 26.76 & 1.11 \\
158 & 123052.28 & 122059.9 & 26.77 & 2.58 \\
159 & 123034.65 & 122553.8 & 26.77 & 4.76 \\
160 & 123057.25 & 122243.5 & 26.77 & 2.30 \\
161 & 123035.64 & 123022.7 & 26.77 & 7.69 \\
162 & 123049.20 & 122151.6 & 26.79 & 1.65 \\
163 & 123036.91 & 122407.3 & 26.79 & 3.70 \\
164 & 123104.55 & 122458.2 & 26.80 & 5.24 \\
165 & 123053.75 & 122804.2 & 26.80 & 5.26 \\
166 & 123040.16 & 122708.9 & 26.80 & 4.35 \\
167 & 123045.69 & 122904.7 & 26.81 & 5.79 \\
168 & 123053.15 & 122055.9 & 26.81 & 2.70 \\
169 & 123040.19 & 122715.1 & 26.81 & 4.43 \\
170 & 123109.26 & 122708.7 & 26.81 & 7.84 \\
171 & 123041.48 & 122303.5 & 26.82 & 2.31
\end{tabular}


Table 1-Continued

\begin{tabular}{|c|c|c|c|c|}
\hline ID & $\alpha(2000)$ & $\delta(2000)$ & $m_{5007}$ & $R_{\text {iso }}$ \\
\hline 172 & 123046.38 & 122313.5 & 26.83 & 0.79 \\
\hline 173 & 123045.89 & 122438.4 & 26.83 & 1.44 \\
\hline 174 & 123117.38 & 122207.6 & 26.83 & 8.52 \\
\hline 175 & 123042.72 & 122529.2 & 26.83 & 2.62 \\
\hline 176 & 123051.83 & 122348.9 & 26.84 & 0.78 \\
\hline 177 & 123040.20 & 122530.1 & 26.83 & 3.16 \\
\hline 178 & 123042.33 & 122054.4 & 26.84 & 3.67 \\
\hline 179 & 123047.58 & 122554.1 & 26.84 & 2.53 \\
\hline 180 & 123046.84 & 122409.8 & 26.85 & 0.93 \\
\hline 181 & 123040.92 & 122318.8 & 26.85 & 2.41 \\
\hline 182 & 123027.24 & 122054.5 & 26.86 & 7.72 \\
\hline 183 & 123053.62 & 122749.9 & 26.86 & 5.00 \\
\hline 184 & 123100.71 & 122914.1 & 26.86 & 7.54 \\
\hline 185 & 123108.95 & 122317.3 & 26.86 & 6.04 \\
\hline 186 & 123059.94 & 122241.5 & 26.87 & 3.16 \\
\hline 187 & 123045.22 & 122628.2 & 26.89 & 3.18 \\
\hline 188 & 123033.98 & 122850.9 & 26.89 & 6.66 \\
\hline 189 & 123047.07 & 122409.9 & 26.89 & 0.89 \\
\hline 190 & 123047.71 & 122412.7 & 26.89 & 0.85 \\
\hline 191 & 123051.46 & 122137.0 & 26.89 & 1.92 \\
\hline 192 & 123057.89 & 122450.6 & 26.89 & 3.15 \\
\hline 193 & 123041.24 & 122410.9 & 26.89 & 2.30 \\
\hline 194 & 123045.74 & 122427.8 & 26.90 & 1.33 \\
\hline
\end{tabular}


Table 1-Continued

\begin{tabular}{cccccc}
\hline \hline & & & & \\
ID & $\alpha(2000)$ & $\delta(2000)$ & $m_{5007}$ & $R_{\text {iso }}$ \\
\hline & & & & \\
195 & 123039.47 & 122550.6 & 26.91 & 3.53 \\
196 & 123040.12 & 122816.6 & 26.91 & 5.32 \\
197 & 123052.23 & 122429.8 & 26.92 & 1.42 \\
198 & 123057.80 & 122725.6 & 26.92 & 5.31 \\
199 & 123045.08 & 122256.1 & 26.92 & 1.29 \\
200 & 123047.84 & 122551.4 & 26.93 & 2.48 \\
201 & 123042.52 & 121829.8 & 26.93 & 5.92 \\
202 & 123057.62 & 122543.7 & 26.93 & 3.79 \\
203 & 123042.37 & 122537.9 & 26.94 & 2.79 \\
204 & 123053.75 & 121719.8 & 26.94 & 6.31 \\
205 & 123052.60 & 122701.7 & 26.95 & 4.05 \\
206 & 123023.10 & 122634.7 & 26.95 & 8.12 \\
207 & 123045.66 & 122559.5 & 26.96 & 2.69 \\
208 & 123038.43 & 122225.2 & 26.96 & 3.63 \\
209 & 123043.14 & 121806.4 & 26.98 & 6.22 \\
210 & 123100.86 & 122302.5 & 26.98 & 3.46 \\
211 & 123104.02 & 122154.0 & 26.98 & 4.55 \\
212 & 123046.47 & 122744.9 & 26.98 & 4.42 \\
213 & 123109.30 & 121659.2 & 26.99 & 8.30 \\
214 & 123042.36 & 121532.7 & 26.99 & 8.91 \\
215 & 123051.93 & 121802.4 & 27.00 & 5.57 \\
216 & 123018.53 & 122413.1 & 27.00 & 9.35 \\
217 & 123047.57 & 122140.5 & 27.00 & 1.96
\end{tabular}


Table 1-Continued

\begin{tabular}{cccccc}
\hline \hline & & & & \\
ID & $\alpha(2000)$ & $\delta(2000)$ & $m_{5007}$ & $R_{\text {iso }}$ \\
\hline 218 & 123042.16 & 122028.6 & 27.00 & 4.11 \\
219 & 123043.92 & 121930.3 & 27.00 & 4.71 \\
220 & 123108.61 & 122739.8 & 27.00 & 8.03 \\
221 & 123037.94 & 122543.3 & 27.01 & 3.83 \\
222 & 123054.42 & 122448.5 & 27.00 & 2.17 \\
223 & 123052.87 & 122114.8 & 27.01 & 2.38 \\
224 & 123052.64 & 122530.6 & 27.01 & 2.49 \\
225 & 123032.08 & 122056.9 & 27.01 & 6.31 \\
226 & 123044.07 & 122354.1 & 27.02 & 1.44 \\
227 & 123051.42 & 122654.0 & 27.03 & 3.78 \\
228 & 123118.45 & 121813.9 & 27.03 & 9.64 \\
229 & 123027.84 & 121702.2 & 27.03 & 0.23 \\
230 & 123040.40 & 122052.0 & 27.04 & 4.17 \\
231 & 123050.66 & 122506.7 & 27.05 & 1.81 \\
232 & 123044.64 & 122149.5 & 27.05 & 2.29 \\
233 & 123042.22 & 122216.2 & 27.05 & 2.53 \\
234 & 123100.21 & 122515.8 & 27.05 & 4.16 \\
235 & 123032.33 & 122446.1 & 27.05 & 5.15 \\
236 & 123053.71 & 122137.4 & 27.05 & 2.13 \\
237 & 123031.93 & 121819.3 & 27.06 & 8.23 \\
238 & 123044.55 & 122639.1 & 27.07 & 3.40 \\
239 & 123023.23 & 122003.9 & 27.07 & 9.33 \\
240 & 123057.69 & 122844.6 & 27.08 & 6.53
\end{tabular}


Table 1-Continued

\begin{tabular}{ccccc}
\hline \hline & & & & \\
ID & $\alpha(2000)$ & $\delta(2000)$ & $m_{5007}$ & $R_{\text {iso }}$ \\
\hline & & & & \\
241 & 123032.47 & 122146.2 & 27.08 & 5.75 \\
242 & 123050.90 & 122057.3 & 27.08 & 2.58 \\
243 & 123056.88 & 122118.6 & 27.09 & 2.91 \\
244 & 123029.30 & 122612.7 & 27.09 & 6.30 \\
245 & 123044.09 & 122330.0 & 27.09 & 1.40 \\
246 & 123027.31 & 122133.2 & 27.10 & 7.38 \\
247 & 123056.39 & 122311.0 & 27.10 & 1.98 \\
248 & 123041.04 & 122549.4 & 27.10 & 3.18 \\
249 & 123046.16 & 122236.2 & 27.11 & 1.26 \\
250 & 123052.82 & 122705.2 & 27.11 & 4.14 \\
251 & 123041.77 & 122336.3 & 27.11 & 2.09 \\
252 & 123047.16 & 121913.1 & 27.11 & 4.59 \\
253 & 123038.27 & 122458.8 & 27.12 & 3.42 \\
254 & 123102.85 & 121938.8 & 27.12 & 5.24 \\
255 & 123044.92 & 122556.1 & 27.12 & 2.70 \\
256 & 123057.73 & 122405.4 & 27.13 & 2.65 \\
257 & 123047.52 & 122007.9 & 27.13 & 3.60 \\
258 & 123039.93 & 122139.4 & 27.13 & 3.66 \\
259 & 123110.83 & 122034.8 & 27.14 & 6.78 \\
260 & 123057.80 & 122542.6 & 27.14 & 3.82 \\
261 & 123044.10 & 122209.8 & 27.14 & 2.11 \\
262 & 123053.98 & 122139.8 & 27.15 & 2.14 \\
263 & 123044.74 & 122141.9 & 27.15 & 2.39
\end{tabular}


Table 1-Continued

\begin{tabular}{lccccc}
\hline \hline & & & & \\
ID & $\alpha(2000)$ & $\delta(2000)$ & $m_{5007}$ & $R_{\text {iso }}$ \\
\hline & & & & \\
264 & 123032.45 & 121904.3 & 27.16 & 7.51 \\
265 & 123039.26 & 122459.7 & 27.16 & 3.14 \\
266 & 123056.74 & 122125.4 & 27.17 & 2.80 \\
267 & 123042.39 & 122541.5 & 27.17 & 2.83 \\
268 & 123058.42 & 121955.4 & 27.17 & 4.23 \\
269 & 123105.60 & 122359.9 & 27.17 & 5.17 \\
270 & 123057.87 & 122442.5 & 27.18 & 3.05 \\
271 & 123054.61 & 121543.5 & 27.18 & 7.96 \\
272 & 123057.72 & 122215.4 & 27.18 & 2.58 \\
273 & 123043.29 & 122555.8 & 27.18 & 2.88 \\
274 & 123039.57 & 122335.1 & 27.19 & 2.82 \\
275 & 123055.00 & 121824.3 & 27.19 & 5.26 \\
276 & 123045.78 & 122248.5 & 27.20 & 1.19 \\
277 & 123050.41 & 121943.2 & 27.20 & 3.86 \\
278 & 123029.59 & 122801.4 & 27.21 & 7.00 \\
279 & 123035.80 & 121851.7 & 27.21 & 6.89 \\
280 & 123054.42 & 121628.2 & 27.21 & 7.20 \\
281 & 123110.11 & 121748.9 & 27.22 & 7.90 \\
282 & 123108.18 & 121924.5 & 27.22 & 6.56 \\
283 & 123025.64 & 121943.5 & 27.22 & 8.85 \\
284 & 123058.62 & 122507.3 & 27.22 & 3.58 \\
285 & 123055.38 & 122657.1 & 27.23 & 4.41 \\
286 & 123053.86 & 121621.3 & 27.23 & 7.31
\end{tabular}


Table 1-Continued

\begin{tabular}{ccccc}
\hline \hline & & & & \\
ID & $\alpha(2000)$ & $\delta(2000)$ & $m_{5007}$ & $R_{\text {iso }}$ \\
\hline & & & & \\
287 & 123048.69 & 122151.4 & 27.23 & 1.68 \\
288 & 123052.85 & 122604.6 & 27.23 & 3.09 \\
289 & 123041.12 & 122405.4 & 27.24 & 2.32 \\
290 & 123045.93 & 122120.7 & 27.24 & 2.53 \\
291 & 123108.37 & 122706.6 & 27.25 & 7.58 \\
292 & 123055.14 & 121845.3 & 27.25 & 4.92 \\
293 & 123021.35 & 121803.9 & 27.25 & 1.10 \\
294 & 123041.50 & 121733.7 & 27.25 & 7.01 \\
295 & 123054.53 & 122109.7 & 27.26 & 2.63 \\
296 & 123059.26 & 121928.1 & 27.26 & 4.72 \\
297 & 123100.21 & 122321.9 & 27.27 & 3.27 \\
298 & 123039.13 & 122820.9 & 27.27 & 5.50 \\
299 & 123051.77 & 122629.7 & 27.28 & 3.38 \\
300 & 123044.92 & 121952.0 & 27.28 & 4.20 \\
301 & 123045.89 & 122425.5 & 27.29 & 1.28 \\
302 & 123058.85 & 122325.9 & 27.30 & 2.81 \\
303 & 123039.75 & 122243.5 & 27.31 & 3.03 \\
304 & 123042.79 & 121841.3 & 27.34 & 5.69 \\
305 & 123037.67 & 122137.0 & 27.35 & 4.35 \\
306 & 123100.82 & 121704.0 & 27.36 & 6.99 \\
307 & 123052.95 & 122005.3 & 27.38 & 3.50 \\
308 & 123055.80 & 122116.2 & 27.38 & 2.74 \\
309 & 123025.43 & 122711.9 & 27.39 & 7.67
\end{tabular}


Table 1-Continued

\begin{tabular}{cccccc}
\hline \hline & & & & \\
ID & $\alpha(2000)$ & $\delta(2000)$ & $m_{5007}$ & $R_{\text {iso }}$ \\
\hline 310 & 123050.33 & 121859.3 & 27.39 & 4.62 \\
311 & 123040.34 & 122343.3 & 27.40 & 2.55 \\
312 & 123043.97 & 122035.3 & 27.40 & 3.63 \\
313 & 123041.84 & 122253.6 & 27.41 & 2.27 \\
314 & 123055.58 & 122027.2 & 27.42 & 3.38 \\
315 & 123029.80 & 122207.8 & 27.43 & 6.39 \\
316 & 123109.30 & 121821.9 & 27.43 & 7.39 \\
317 & 123040.75 & 122301.6 & 27.44 & 2.56 \\
318 & 123059.65 & 121931.2 & 27.45 & 4.74 \\
319 & 123059.62 & 122206.1 & 27.45 & 3.19 \\
320 & 123058.68 & 122527.3 & 27.46 & 3.86 \\
321 & 123042.12 & 121846.9 & 27.47 & 5.71 \\
322 & 123048.80 & 122132.5 & 27.52 & 2.00 \\
323 & 123041.55 & 122320.0 & 27.52 & 2.20 \\
324 & 123047.65 & 122545.8 & 27.52 & 2.38 \\
325 & 123056.30 & 122333.8 & 27.52 & 2.00 \\
326 & 123103.60 & 122543.1 & 27.55 & 5.38 \\
327 & 123043.40 & 122126.0 & 27.55 & 2.93 \\
328 & 123100.47 & 122446.9 & 27.56 & 3.94 \\
329 & 123053.33 & 122814.6 & 27.57 & 5.38 \\
330 & 123103.55 & 121856.3 & 27.59 & 5.85 \\
331 & 123040.20 & 121922.9 & 27.60 & 5.49 \\
332 & 123101.50 & 122136.4 & 27.61 & 3.91
\end{tabular}


Table 1-Continued

\begin{tabular}{cccccc}
\hline \hline & & & & & \\
ID & $\alpha(2000)$ & $\delta(2000)$ & $m_{5007}$ & $R_{\text {iso }}$ \\
\hline & & & & & \\
333 & 123046.66 & 122139.1 & 27.61 & 2.10 \\
334 & 123055.99 & 122019.2 & 27.61 & 3.55 \\
335 & 123026.48 & 122013.9 & 27.61 & 8.31 \\
336 & 123039.66 & 122358.4 & 27.64 & 2.77 \\
337 & 123033.19 & 122055.5 & 27.64 & 6.02 \\
338 & 123042.09 & 122420.2 & 27.65 & 2.10 \\
339 & 123048.74 & 122202.5 & 27.86 & 1.48 \\
\hline
\end{tabular}


Table 2. Planetary Nebulae Associated with other galaxies

\begin{tabular}{|c|c|c|c|}
\hline ID & $\alpha(2000)$ & $\delta(2000)$ & $m_{5007}$ \\
\hline NGC $4478-1$ & 123024.48 & 122001.3 & 26.18 \\
\hline NGC $4478-2$ & 123018.52 & 121921.9 & 26.37 \\
\hline NGC $4478-3$ & 123018.45 & 121924.9 & 26.95 \\
\hline NGC $4478-4$ & 123018.60 & 121928.5 & 26.79 \\
\hline NGC $4478-5$ & 123018.48 & 121930.9 & 26.509 \\
\hline NGC $4478-6$ & 123018.57 & 121958.4 & 27.04 \\
\hline NGC $4478-7$ & 123019.10 & 121917.3 & 27.65 \\
\hline IC $3443-1$ & 123113.20 & 121955.2 & 26.73 \\
\hline Anonymous - 1 & 123027.29 & 121654.9 & 26.69 \\
\hline
\end{tabular}


Table 3. PN Photometric Error Versus Magnitude

\begin{tabular}{lcc}
\hline \hline & & \\
Magnitude & Mean $1 \sigma$ error & Number \\
\hline 25.6 & 0.036 & 1 \\
26.0 & 0.050 & 4 \\
26.2 & 0.056 & 12 \\
26.4 & 0.061 & 18 \\
26.6 & 0.080 & 37 \\
26.8 & 0.093 & 40 \\
27.0 & 0.110 & 52 \\
27.2 & 0.125 & 48 \\
27.4 & 0.142 & 21 \\
27.6 & 0.171 & 14 \\
\hline
\end{tabular}




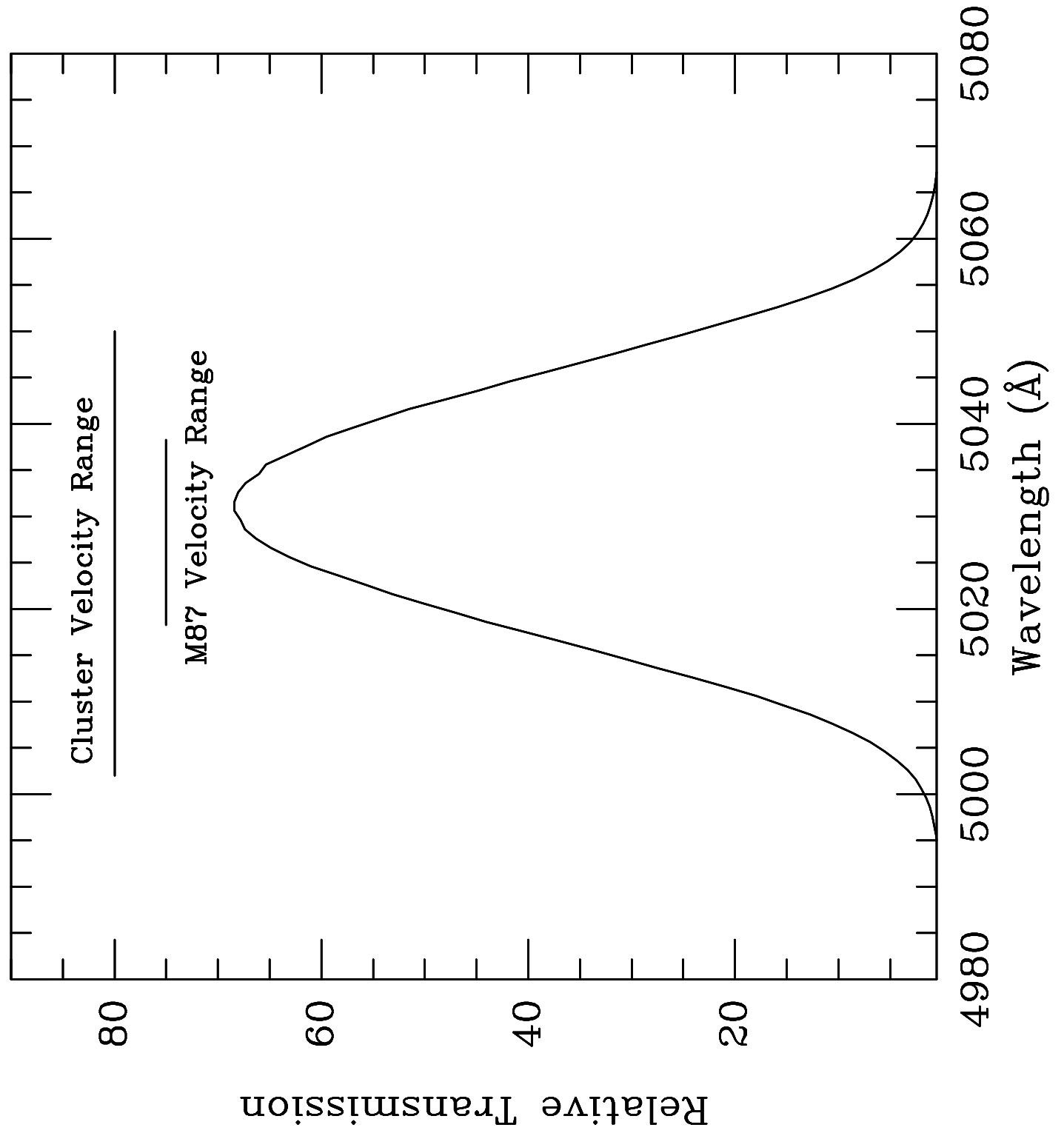




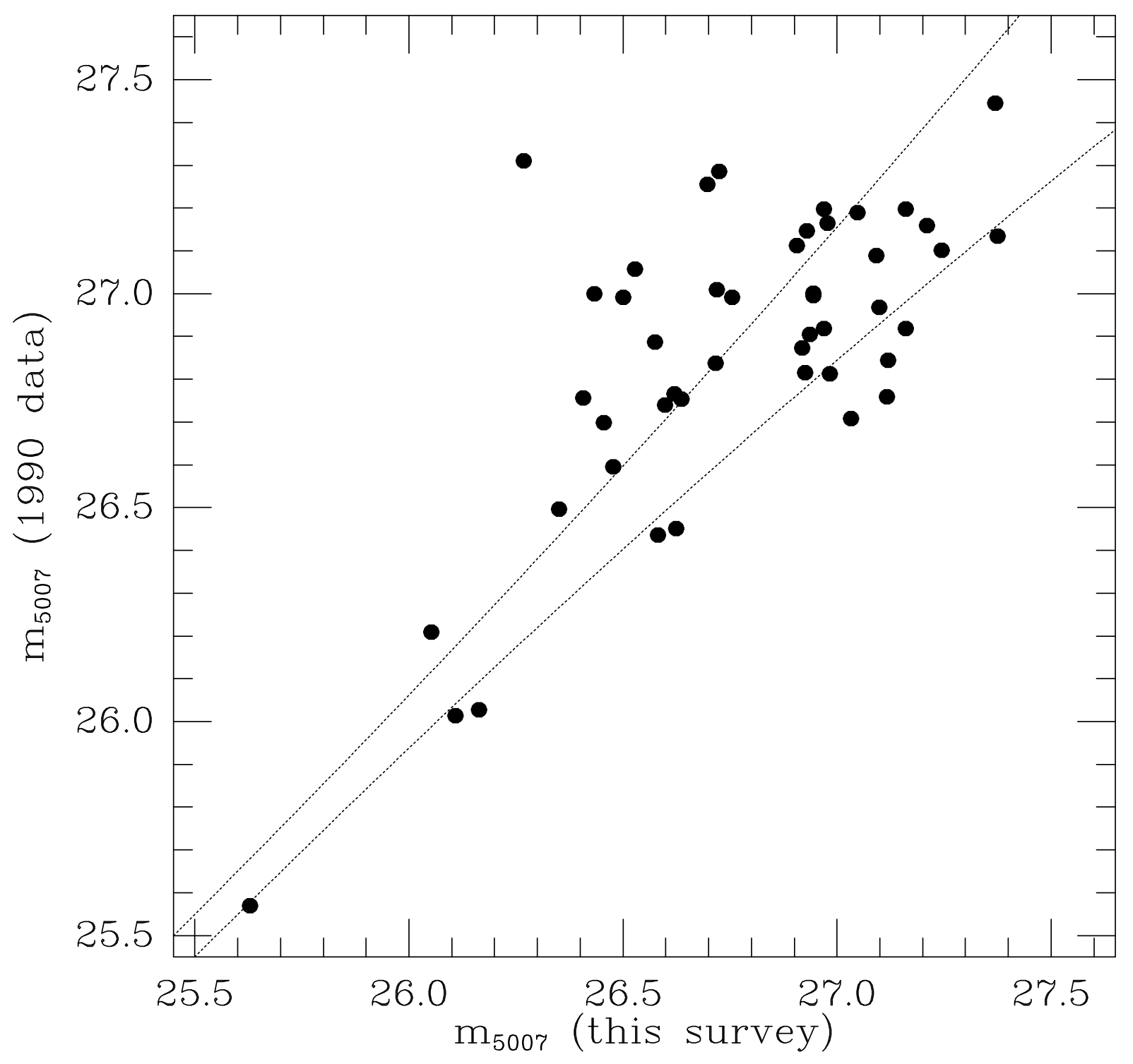




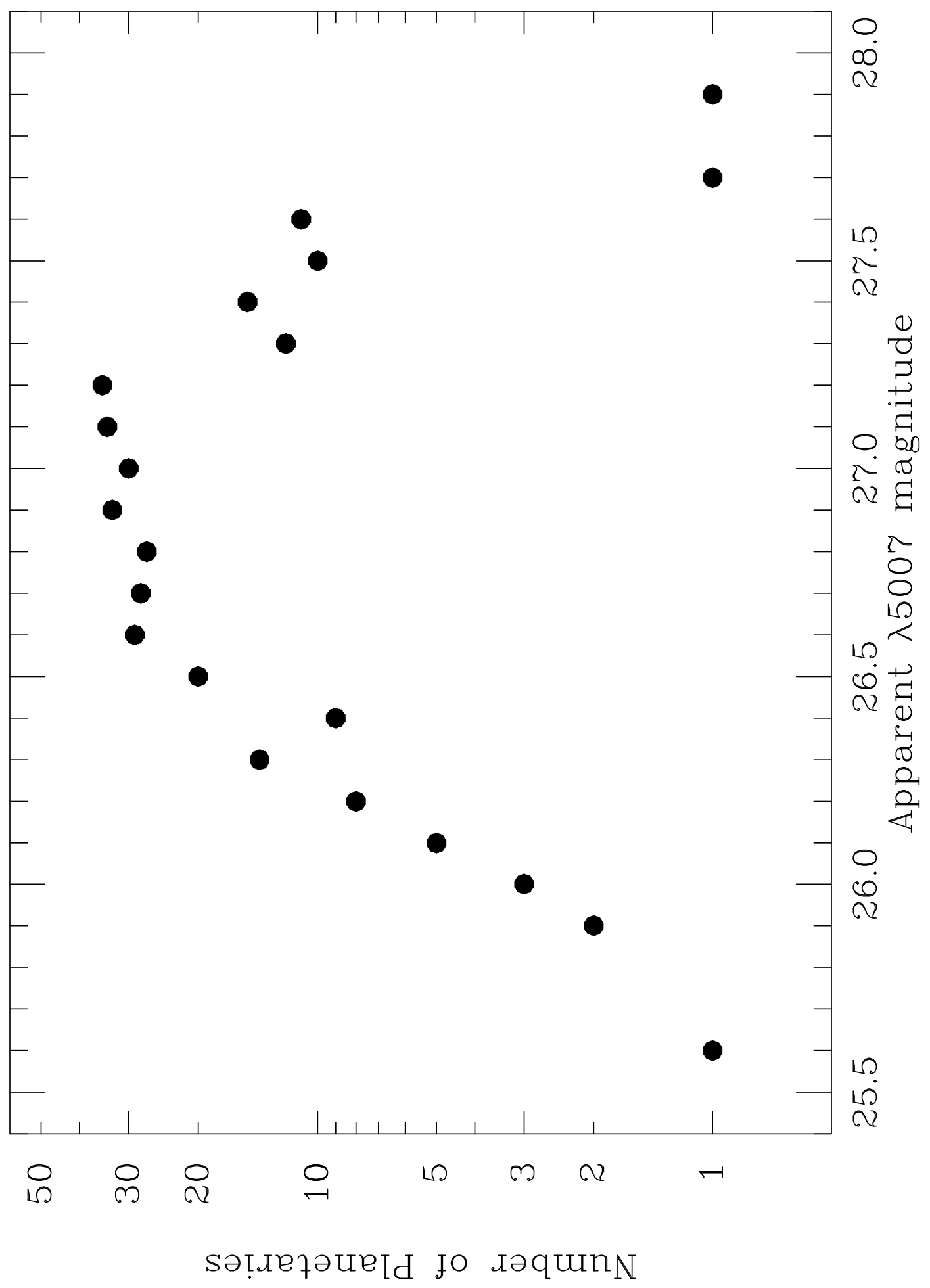




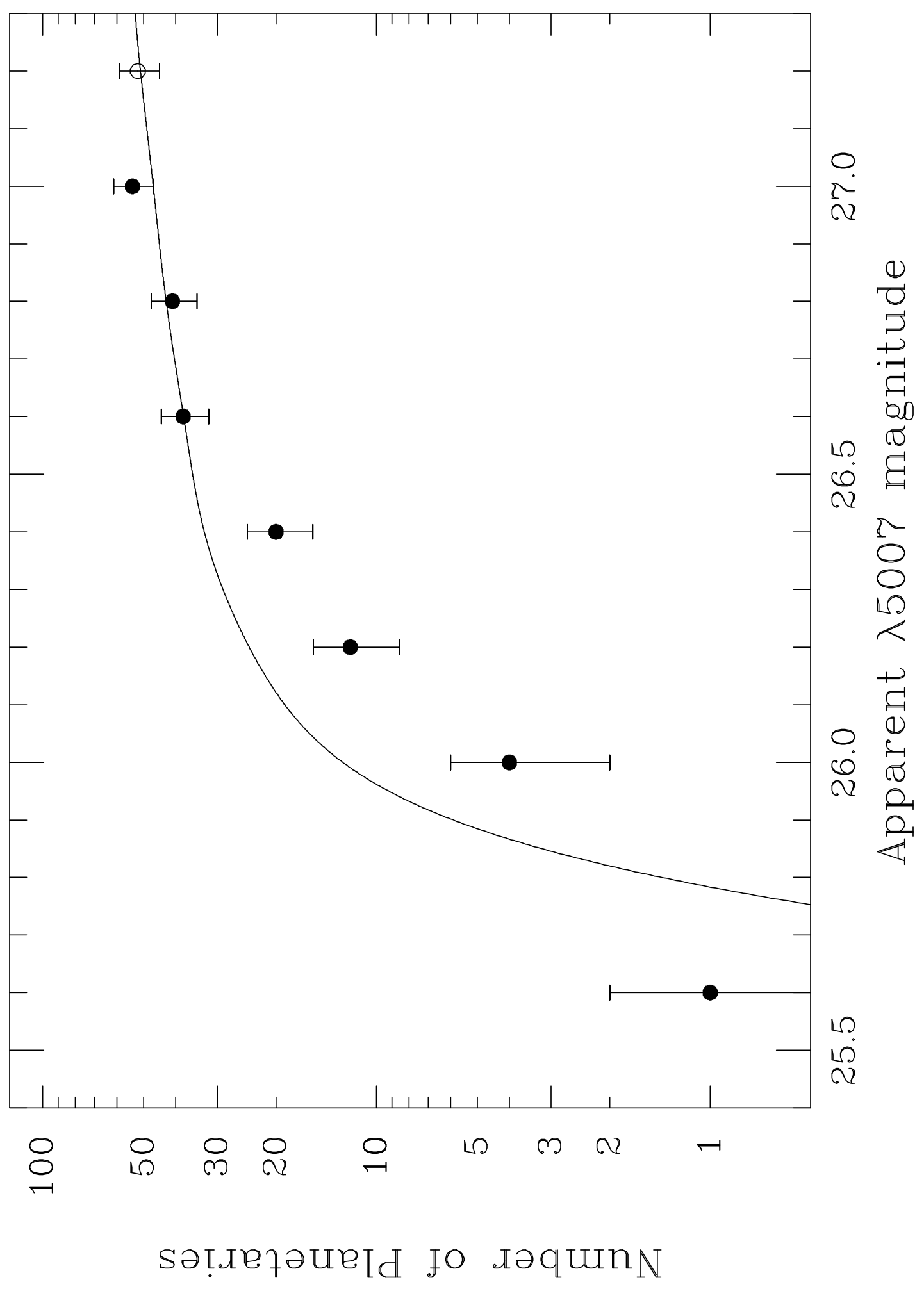




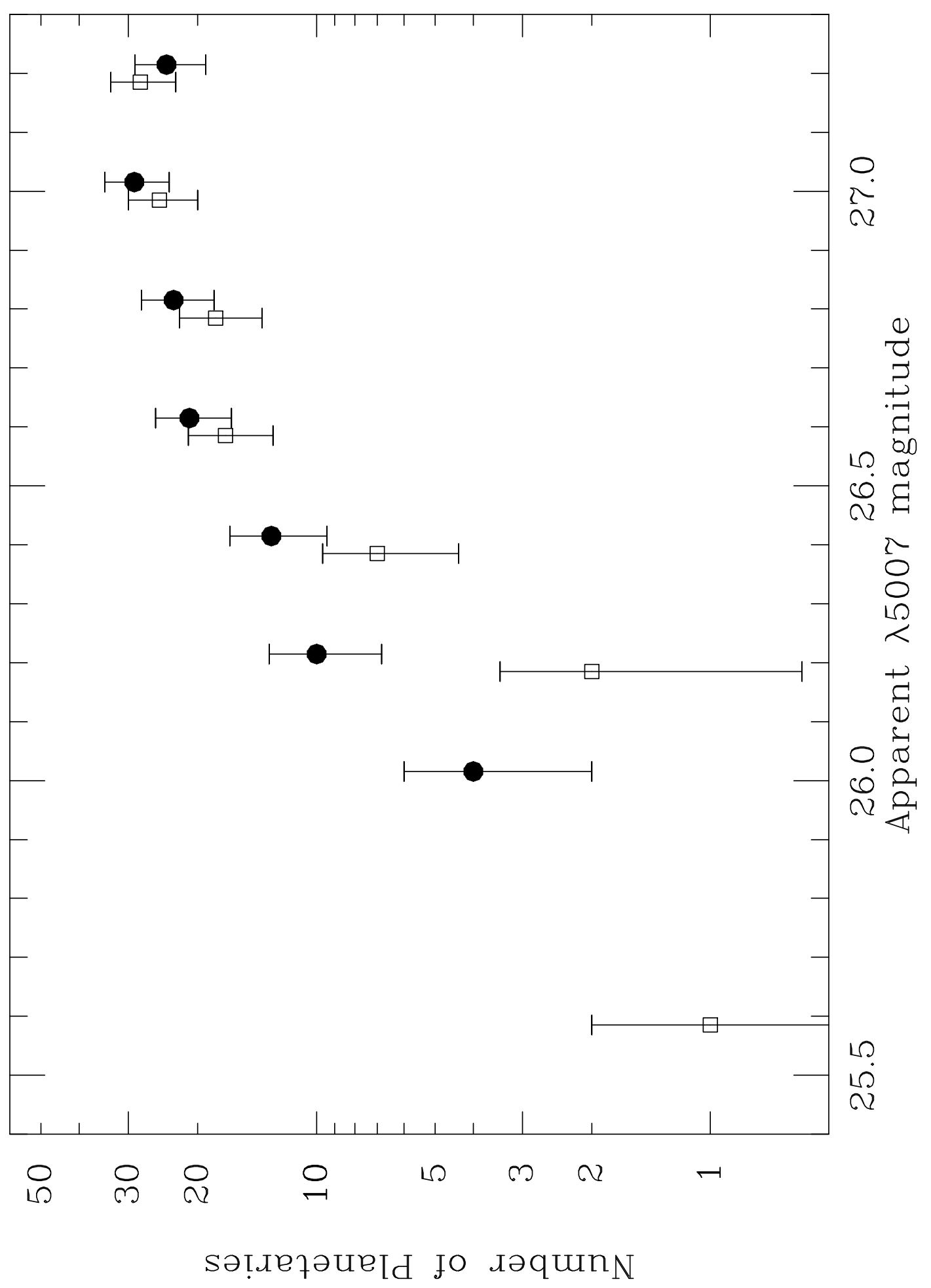




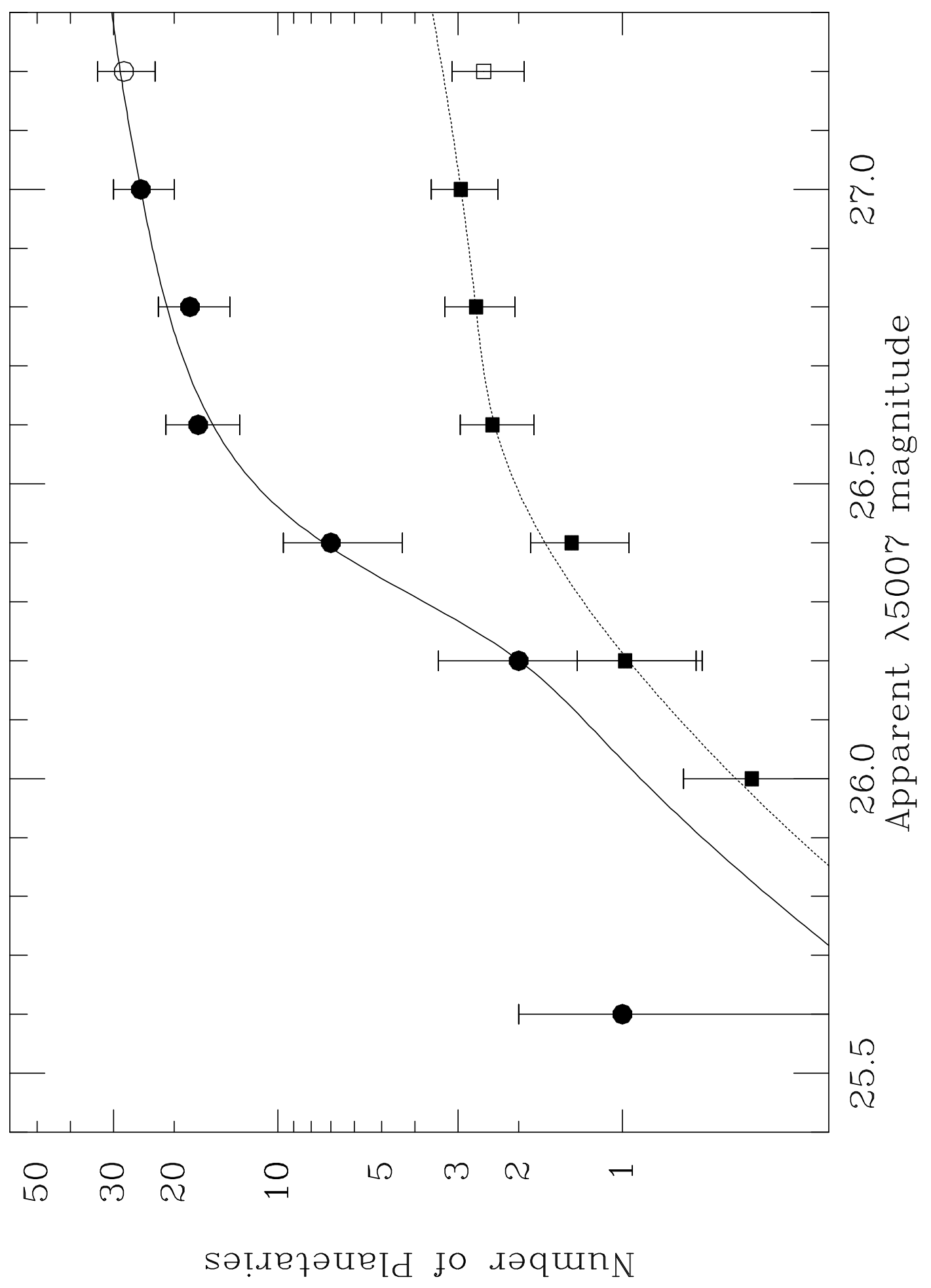




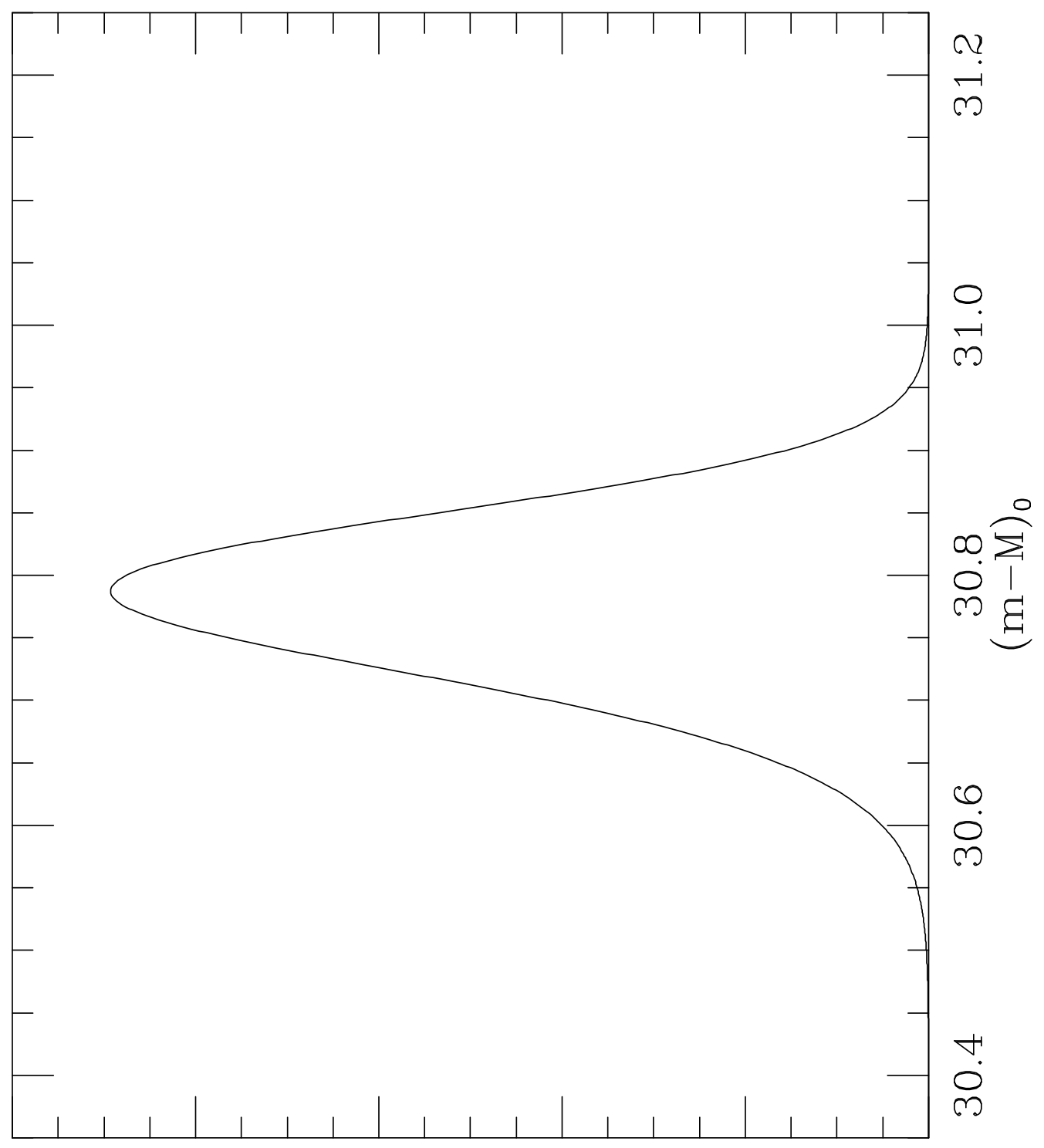

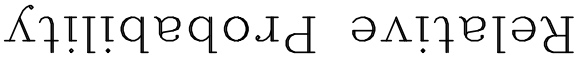




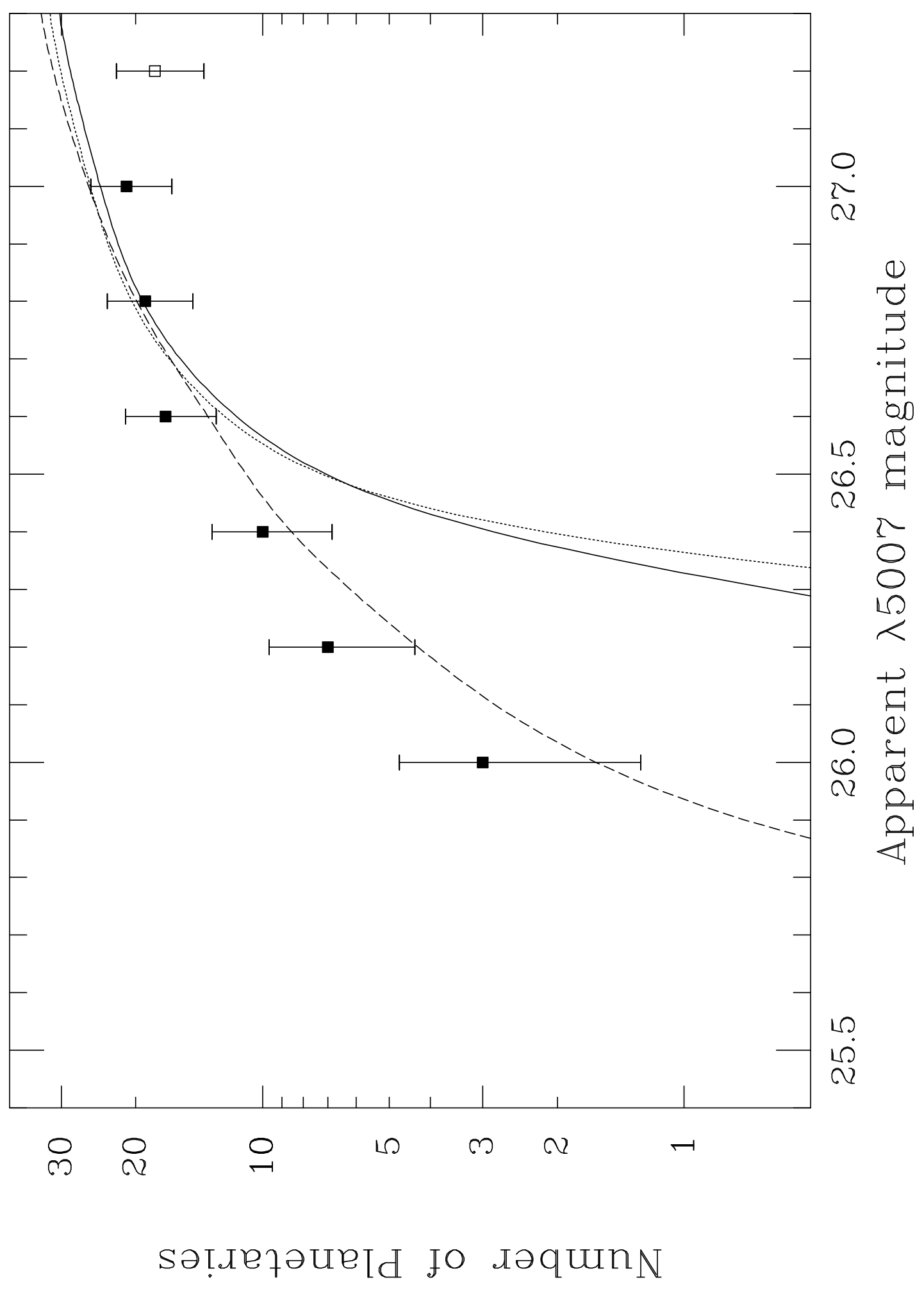

